\title{
Article \\ A Type of Time-Symmetric Stochastic System and Related Games
}

\author{
Qingfeng Zhu ${ }^{1,2,3} \mathbb{D}^{-}$, Yufeng Shi ${ }^{3, *} \mathbb{C}$, Jiaqiang Wen ${ }^{4}$ and Hui Zhang ${ }^{1,2}$ \\ 1 School of Mathematics and Quantitative Economics, Shandong University of Finance and Economics, \\ Jinan 250014, China; qfzhu@sdufe.edu.cn (Q.Z.); zhanghui@sdufe.edu.cn (H.Z.) \\ 2 Shandong Key Laboratory of Blockchain Finance, Shandong University of Finance and Economics, \\ Jinan 250014, China \\ 3 Institute for Financial Studies and School of Mathematics, Shandong University, Jinan 250100, China \\ 4 Department of Mathematics, Southern University of Science and Technology, Shenzhen 518055, China; \\ wenjq@sustech.edu.cn \\ * Correspondence: yfshi@sdu.edu.cn
}

Citation: Zhu, Q.; Shi, Y.; Wen, J.; Zhang, H. A Type of Time-Symmetric Stochastic System and Related Games. Symmetry 2021, 13, 118. https:// doi.org/10.3390/sym13010118

Received: 17 December 2020 Accepted: 6 January 2021 Published: 12 January 2021

Publisher's Note: MDPI stays neutral with regard to jurisdictional clai$\mathrm{ms}$ in published maps and institutional affiliations.

Copyright: (C) 2021 by the authors. Licensee MDPI, Basel, Switzerland. This article is an open access article distributed under the terms and conditions of the Creative Commons Attribution (CC BY) license (https:// creativecommons.org/licenses/by/ $4.0 /)$.

\begin{abstract}
This paper is concerned with a type of time-symmetric stochastic system, namely the socalled forward-backward doubly stochastic differential equations (FBDSDEs), in which the forward equations are delayed doubly stochastic differential equations (SDEs) and the backward equations are anticipated backward doubly SDEs. Under some monotonicity assumptions, the existence and uniqueness of measurable solutions to FBDSDEs are obtained. The future development of many processes depends on both their current state and historical state, and these processes can usually be represented by stochastic differential systems with time delay. Therefore, a class of nonzero sum differential game for doubly stochastic systems with time delay is studied in this paper. A necessary condition for the open-loop Nash equilibrium point of the Pontriagin-type maximum principle are established, and a sufficient condition for the Nash equilibrium point is obtained. Furthermore, the above results are applied to the study of nonzero sum differential games for linear quadratic backward doubly stochastic systems with delay. Based on the solution of FBDSDEs, an explicit expression of Nash equilibrium points for such game problems is established.
\end{abstract}

Keywords: backward doubly stochastic differential equations; stochastic differential game; maximum principle; Nash equilibrium point; time-delayed generator

\section{Introduction}

In 1994, Pardoux and Peng [1] put forward the following backward doubly stochastic differential equations (BDSDEs):

$$
p(t)=\xi+\int_{t}^{T} F(s, p(s), q(s)) \mathrm{d} s+\int_{t}^{T} G(s, p(s), q(s)) \overleftarrow{\mathrm{d}} B(s)-\int_{t}^{T} q(s) \overrightarrow{\mathrm{d}} W(s), 0 \leq t \leq T,
$$

which can be applied to produce a probabilistic expression of certain quasilinear stochastic partial differential equations (SPDEs). Because of its importance to SPDEs, the interest in BDSDEs has increased considerably (see [2-15]). At the same time, the stochastic control problem of backward doubly stochastic systems has been studied extensively (see [16-21]). 
In 2003, Peng and Shi [22] introduced the following time-symmetric fully coupled forward-backward stochastic systems:

$$
\left\{\begin{aligned}
y(t)=x & +\int_{0}^{t} f(s, p(s), y(s), q(s), z(s)) \mathrm{d} s-\int_{0}^{t} z(s) \overleftarrow{\mathrm{d}} B(s) \\
& \quad+\int_{0}^{t} g(s, p(s), y(s), q(s), z(s)) \overrightarrow{\mathrm{d}} W(s) \\
p(t)= & \Phi(y(T))+\int_{t}^{T} F(s, p(s), y(s), q(s), z(s)) \mathrm{d} s-\int_{t}^{T} q(s) \overrightarrow{\mathrm{d}} W(s) \\
& \quad+\int_{t}^{T} G(s, p(s), y(s), q(s), z(s)) \overleftarrow{\mathrm{d}} B(s)
\end{aligned}\right.
$$

which are the so-called forward-backward doubly stochastic differential equations (FBDSDEs). The forward and backward equations in Equation (2) are the BDSDE in Equation (1) with stochastic integrals in different directions. Therefore, the FBDSDE in Equation (2) is established to provide a more general framework of fully coupled forward-backward stochastic differential equations. Under some monotone assumptions, Peng and Shi [22] obtained the unique solvability of FBDSDEs (2). Zhu et al. [23,24] have extended the results in [22] to different dimensions and the weaker monotonic assumptions, and gave the probabilistic interpretation for the solutions to SPDEs combined with algebra equations. Zhang and Shi [25] and Shi and Zhu [26] studied the stochastic control problem of FBDSDEs.

Game theory has penetrated into economic theory and attracted more and more research. It was first proposed by Von Neumann and Morgenstern [27]. Nash [28] has done groundbreaking work on non-cooperative games and presents the concept of a classic Nash equilibrium. Zhao et al. [29] studied the optimal investment and reinsurance of insurers in default securities under a mean-variance criterion in the jump-diffusion risk model. Many papers on stochastic differential game problems driven by backward stochastic differential equations have been published (see [30-32]). The differential game problem for forward-backward doubly stochastic differential equations was addressed in [33]. However, the future evolution of a lot of processes depends not only on their current state, but also on their historical state, and these processes can usually be characterized by stochastic differential equations with time delay. The optimal control problem for stochastic differential equations with delay was discussed in [34-39]. The nonzero sum differential game of the stochastic differential delay equation was studied in [40,41]. Shen and Zeng [42] researched the optimal investment and reinsurance with time delay for insurers under a mean-variance criterion.

The extra noise $\{B(t)\}$ in Equation (1) can be regarded as some additional financial information that is not disclosed to the public in practice, such as in the derivative securities market, but is available to some investors. Arriojas et al. [43] and Kazmerchuk et al. [44] obtained the option pricing formula with time delay based on the stock price process with time delay. As far as we know, there is little discussion about differential games of doubly stochastic systems with delay. In this article, we will discuss this direction, that is, the following nonzero sum differential game driven by doubly stochastic systems with time delay. The control system is

$$
\left\{\begin{aligned}
\mathrm{d} y(t)= & f\left(t, y(t), z(t), y_{\delta}(t), z_{\delta}(t), v_{1}(t), v_{2}(t)\right) \mathrm{d} t-z(t) \overleftarrow{\mathrm{d}} B(t) \\
& +g\left(t, y(t), z(t), y_{\delta}(t), z_{\delta}(t), v_{1}(t), v_{2}(t)\right) \overrightarrow{\mathrm{d}} W(t), t \in[0, T] \\
y(t)= & \phi(t), z(t)=\psi(t), t \in[-\delta, 0]
\end{aligned}\right.
$$

where $(y(\cdot), z(\cdot)) \in \mathbb{R}^{n} \times \mathbb{R}^{n \times d}$ is the state process pair, $0<\delta<T$ is a constant time delay parameter, and $y_{\delta}(t)=y(t-\delta), z_{\delta}(t)=z(t-\delta)$. We denote $J_{1}(v(\cdot))$ and $J_{2}(v(\cdot))$, $v(\cdot)=\left(v_{1}(\cdot), v_{2}(\cdot)\right)$, which are the cost functionals corresponding to the players 1 and 2 :

$J_{i}\left(v_{1}(\cdot), v_{2}(\cdot)\right)=\mathbb{E}\left\{\int_{0}^{T} l_{i}\left(t, y(t), z(t), y_{\delta}(t), z_{\delta}(t), v_{1}(t), v_{2}(t)\right) \mathrm{d} t+\Phi_{i}(y(T))\right\}, i=1,2$. 
Our task is to find $\left(u_{1}(\cdot), u_{2}(\cdot)\right) \in \mathcal{U}_{1} \times \mathcal{U}_{2}$ such that

$$
\left\{\begin{array}{l}
J_{1}\left(u_{1}(\cdot), u_{2}(\cdot)\right)=\min _{v_{1}(\cdot) \in \mathcal{U}_{1}} J_{1}\left(v_{1}(\cdot), u_{2}(\cdot)\right), \\
J_{2}\left(u_{1}(\cdot), u_{2}(\cdot)\right)=\min _{v_{2}(\cdot) \in \mathcal{U}_{2}} J_{2}\left(u_{1}(\cdot), v_{2}(\cdot)\right) .
\end{array}\right.
$$

To figure out the above nonzero sum differential game problem, it is natural to involve the adjoint equation, which is a kind of anticipated BDSDE (see $[45,46]$ ). It is therefore necessary to explore the following general FBDSDE with the forward equation being a delayed doubly SDE and the backward equation being the anticipated BDSDE:

$$
\left\{\begin{aligned}
\mathrm{d} y(t)= & f\left(t, y(t), p(t), z(t), q(t), y_{\delta}(t), z_{\delta}(t)\right) \mathrm{d} t-z(t) \overleftarrow{\mathrm{d}} B(t) \\
& +g\left(t, y(t), p(t), z(t), q(t), y_{\delta}(t), z_{\delta}(t)\right) \overrightarrow{\mathrm{d}} W(t), t \in[0, T], \\
-\mathrm{d} p(t)= & F\left(t, y(t), p(t), z(t), q(t), p_{\delta+}(t), q_{\delta+}(t)\right) \mathrm{d} t-q(t) \overrightarrow{\mathrm{d}} W(t) \\
& +G\left(t, y(t), p(t), z(t), q(t), p_{\delta+}(t), q_{\delta+}(t)\right) \overleftarrow{\mathrm{d}} B(t), t \in[0, T], \\
y(t)= & \phi(t), t \in[-\delta, 0], \\
z(t)= & \psi(t), t \in[-\delta, 0], \\
p(T)= & \Phi(y(T)), p(t)=\xi(t), t \in[T, T+\delta], \\
q(t)= & \eta(t), t \in[T, T+\delta],
\end{aligned}\right.
$$

where $y_{\delta}(t)=y(t-\delta), z_{\delta}=z(t-\delta), p_{\delta+}(t)=p(t+\delta), q_{\delta+}=q(t+\delta)$.

Our work differs from the above in the following distinctions. First of all, we introduce a time-symmetric stochastic system, which generalizes the results in [22] to a more general case: forward doubly stochastic differential equations (SDEs) with delay as forward equations and anticipated backward doubly stochastic differential equations as backward equations. Secondly, we investigate the problem of a nonzero sum differential game driven by doubly stochastic systems with time delay, which enriches the types of stochastic delayed differential game problems. Finally, we explore the linear quadratic (LQ) games for a doubly stochastic system with time delay, and use the solution of the above general FBDSDE to give an explicit expression of the unique equilibrium point.

The structure of this paper is as follows. We give the framework of the doubly stochastic games with delay and a preliminary view on the general FBDSDE in Section 2. We set up a necessary condition for the open-loop Nash equilibrium of such games to form a Pontryagin maximum principle in Section 3. Section 4 is devoted to the verification theorem of a sufficient condition for Nash equilibrium. In order to visually demonstrate the above results, the nonzero sum differential game for LQ double stochastic delay systems is studied in Section 5. By using the results of our FBDSDE, the explicit representation of Nash equilibrium points for LQ game problems is obtained. For the convenience of the reader, we present the skeleton of the proof on uniqueness and existence for the general FBDSDE in Section 6. Finally, we conclude this article with a summary.

\section{Formulation of Problems and Preliminaries}

\subsection{Notations and Formulation of Problems}

Suppose $(\Omega, \mathcal{F}, P)$ is a probability space, and $[0, T]$ is a fixed arbitrarily large time duration throughout this paper. Let $\{W(t) ; 0 \leq t \leq T\}$ and $\{B(t) ; 0 \leq t \leq T\}$ be two mutually independent standard Brownian motions defined on $(\Omega, \mathcal{F}, P)$, with values in $\mathbb{R}^{d}$ and $\mathbb{R}^{l}$, respectively. Let $\mathcal{N}$ denote the class of $P$-null elements of $\mathcal{F}$. For each $t \in[0, T]$, we define $\mathcal{F}_{t} \doteq \mathcal{F}_{t}^{W} \vee \mathcal{F}_{t, T}^{B}$, where $\mathcal{F}_{t}^{W}=\mathcal{N} \vee \sigma\{W(r)-W(0) ; 0 \leq r \leq t\}$, $\mathcal{F}_{t, T}^{B}=\mathcal{N} \vee \sigma\{B(T)-B(r) ; t \leq r \leq T\}$. Note that the collection $\left\{\mathcal{F}_{t}, t \in[0, T]\right\}$ is neither increasing nor decreasing, and it does not produce a filtration. $\mathbb{E}$ denotes the expectation on $(\Omega, \mathcal{F}, P) . \mathbb{E}^{\mathcal{F}_{t}}:=\mathbb{E}\left[\cdot \mid \mathcal{F}_{t}\right]$ denotes the conditional expectation under $\mathcal{F}_{t}$. We use the usual inner product $\langle\cdot, \cdot\rangle$ and Euclidean norm $|\cdot|$ in $\mathbb{R}^{n}, \mathbb{R}^{m}, \mathbb{R}^{m \times l}$ and $\mathbb{R}^{n \times d}$. The symbol " $\top^{\prime \prime}$ that appears on the superscript indicates the transpose of the matrix. All the equations and 
inequalities mentioned in this paper are in the sense of $\mathrm{d} t \times \mathrm{d} P$ almost surely on $[0, T] \times \Omega$. We introduce the following notations:

$L^{2}\left(\mathcal{F}_{T} ; \mathbb{R}^{n}\right)=\left\{\xi: \xi\right.$ is an $\mathbb{R}^{n}$-valued, $\mathcal{F}_{T}$-measurable random variable s.t. $\left.\mathbb{E}|\xi|^{2}<\infty\right\}$, $L_{\mathcal{F}}^{2}\left(0, T ; \mathbb{R}^{n}\right)=\left\{v(t), 0 \leq t \leq T: v(t)\right.$ is an $\mathbb{R}^{n}$-valued, $\mathcal{F}_{t}$-measurable process

$$
\text { s.t. } \left.\mathbb{E} \int_{0}^{T}|v(t)|^{2} \mathrm{~d} t<\infty\right\} \text {. }
$$

We take into account the following controlled doubly stochastic differential systems with delay:

$$
\left\{\begin{aligned}
\mathrm{d} y(t)= & f\left(t, y(t), z(t), y_{\delta}(t), z_{\delta}(t), v_{1}(t), v_{2}(t)\right) \mathrm{d} t-z(t) \overleftarrow{\mathrm{d}} B(t) \\
& +g\left(t, y(t), z(t), y_{\delta}(t), z_{\delta}(t), v_{1}(t), v_{2}(t)\right) \overrightarrow{\mathrm{d}} W(t), t \in[0, T] \\
y(t)= & \phi(t), z(t)=\psi(t), t \in[-\delta, 0]
\end{aligned}\right.
$$

where $(y(\cdot), z(\cdot)) \in \mathbb{R}^{n} \times \mathbb{R}^{n \times d}$ is the state process pair, $0<\delta<T$ is a constant time delay parameter, and $y_{\delta}(t)=y(t-\delta), z_{\delta}(t)=z(t-\delta)$. Here, $f:[0, T] \times \mathbb{R}^{n} \times \mathbb{R}^{n \times d} \times$ $\mathbb{R}^{n} \times \mathbb{R}^{n \times d} \times \mathbb{R}^{k_{1}} \times \mathbb{R}^{k_{2}} \rightarrow \mathbb{R}^{n}, g:[0, T] \times \mathbb{R}^{n} \times \mathbb{R}^{n \times d} \times \mathbb{R}^{n} \times \mathbb{R}^{n \times d} \times \mathbb{R}^{k_{1}} \times \mathbb{R}^{k_{2}} \rightarrow \mathbb{R}^{n \times l}$ are given functions, and $\phi(\cdot), \psi(\cdot) \in L_{\mathcal{F}}^{2}\left(-\delta, 0 ; \mathbb{R}^{n}\right)$ are the initial paths of $y, z$, respectively.

Let $U_{i}$ be a nonempty convex subset of $\mathbb{R}^{i}$ and $v_{i}(\cdot)$ be the control process of player $i, i=1,2$. We denote by $\mathcal{U}_{i}$ the set of $U_{i}$-valued control processes $v_{i} \in L_{\mathcal{F}}^{2}\left(0, T ; \mathbb{R}^{k_{i}}\right)$ and it is called the admissible control set for player $i, i=1,2$. Each element of $\mathcal{U}_{i}$ is called an (open-loop) admissible control for player $i, i=1$, 2. In addition, $\mathcal{U}=\mathcal{U}_{1} \times \mathcal{U}_{2}$ is called the set of admissible controls for the two players.

We assume that

Hypothesis 1 (H1). $f$ and $g$ are continuously differentiable with respect to $\left(y, y_{\delta}, z, z_{\delta}, v_{1}, v_{2}\right)$, and their partial derivatives are bounded.

Now, if both $v_{1}(\cdot)$ and $v_{2}(\cdot)$ are admissible controls, and assumption (H1) holds, then doubly stochastic differential equation with delay (3) admits a unique solution $(y(\cdot), z(\cdot)) \in$ $L_{\mathcal{F}}^{2}\left(-\delta, T ; \mathbb{R}^{n}\right) \times L_{\mathcal{F}}^{2}\left(-\delta, T ; \mathbb{R}^{n \times l}\right)$ (see [20]). The two players have their own benefits, which are described by the cost functional

$$
J_{i}\left(v_{1}(\cdot), v_{2}(\cdot)\right)=\mathbb{E}\left\{\int_{0}^{T} l_{i}\left(t, y(t), z(t), y_{\delta}(t), z_{\delta}(t), v_{1}(t), v_{2}(t)\right) \mathrm{d} t+\Phi_{i}(y(T))\right\},
$$

where $l_{i}:[0, T] \times \mathbb{R}^{n} \times \mathbb{R}^{n \times d} \times \mathbb{R}^{n} \times \mathbb{R}^{n \times d} \times \mathbb{R}^{k_{1}} \times \mathbb{R}^{k_{2}} \rightarrow \mathbb{R}$ and $\Phi_{i}: \mathbb{R}^{n} \rightarrow \mathbb{R}$ are given functions, $i=1,2$.

We also assume

Hypothesis 2 (H2). $l_{i}$ is continuously differentiable in $\left(y, z, y_{\delta}, z_{\delta}, v_{1}, v_{2}\right)$, its partial derivatives are continuous in $\left(y, z, y_{\delta}, z_{\delta}, v_{1}, v_{2}\right)$ and bounded by $c\left(1+|y|+|z|+\left|y_{\delta}\right|+\left|z_{\delta}\right|+\left|v_{1}\right|+\left|v_{2}\right|\right)$. Moreover, $\Phi_{i}(y)$ is continuously differentiable in $y$ and $\Phi_{i y}(y)$ is bounded by $c(1+|y|)$.

Assume that each participant wants to minimize her /his cost functional $J_{i}\left(v_{1}(\cdot), v_{2}(\cdot)\right)$ by selecting an appropriate admissible control $v_{i}(\cdot)(i=1,2)$. Then the problem is to find a pair of admissible controls $\left(u_{1}(\cdot), u_{2}(\cdot)\right) \in \mathcal{U}_{1} \times \mathcal{U}_{2}$ such that

$$
\left\{\begin{aligned}
J_{1}\left(u_{1}(\cdot), u_{2}(\cdot)\right) & =\min _{v_{1}(\cdot) \in \mathcal{U}_{1}} J_{1}\left(v_{1}(\cdot), u_{2}(\cdot)\right) \\
J_{2}\left(u_{1}(\cdot), u_{2}(\cdot)\right) & =\min _{v_{2}(\cdot) \in \mathcal{U}_{2}} J_{2}\left(u_{1}(\cdot), v_{2}(\cdot)\right)
\end{aligned}\right.
$$


We call the above problem a doubly stochastic differential game with time delay. For simplicity's sake, let us write it as Problem (A). If we can find an admissible control $u(\cdot)=\left(u_{1}(\cdot), u_{2}(\cdot)\right)$ satisfying Equation (4), then we call it an equilibrium point of Problem (A) and denote the corresponding state trajectory by $(y(\cdot), z(\cdot))=\left(y^{u}(\cdot), z^{u}(\cdot)\right)$.

\subsection{The General FBDSDE}

We deal with the following general FBDSDE, in which the forward equation is a delayed doubly SDE, and the backward equation is the anticipated BDSDE:

$$
\left\{\begin{aligned}
\mathrm{d} y(t)= & f\left(t, y(t), p(t), z(t), q(t), y_{\delta}(t), z_{\delta}(t)\right) \mathrm{d} t-z(t) \overleftarrow{\mathrm{d}} B(t) \\
& +g\left(t, y(t), p(t), z(t), q(t), y_{\delta}(t), z_{\delta}(t)\right) \overrightarrow{\mathrm{d}} W(t), t \in[0, T], \\
-\mathrm{d} p(t)= & F\left(t, y(t), p(t), z(t), q(t), p_{\delta+}(t), q_{\delta+}(t)\right) \mathrm{d} t-q(t) \overrightarrow{\mathrm{d}} W(t) \\
& +G\left(t, y(t), p(t), z(t), q(t), p_{\delta+}(t), q_{\delta+}(t)\right) \overleftarrow{\mathrm{d}} B(t), t \in[0, T], \\
y(t)= & \phi(t), t \in[-\delta, 0], \\
z(t)= & \psi(t), t \in[-\delta, 0], \\
p(T)= & \Phi(y(T)), p(t)=\xi(t), t \in[T, T+\delta], \\
q(t)= & \eta(t), t \in[T, T+\delta],
\end{aligned}\right.
$$

where $y_{\delta}(t)=y(t-\delta), z_{\delta}=z(t-\delta), p_{\delta+}(t)=p(t+\delta), q_{\delta+}=q(t+\delta)$, and

$$
\begin{array}{lll}
F & : \Omega \times[0, T] \times \mathbb{R}^{n} \times \mathbb{R}^{m} \times \mathbb{R}^{n \times l} \times \mathbb{R}^{m \times d} \times \mathbb{R}^{m} \times \mathbb{R}^{m \times d} \rightarrow \mathbb{R}^{m}, \\
f & : \Omega \times[0, T] \times \mathbb{R}^{n} \times \mathbb{R}^{m} \times \mathbb{R}^{n \times l} \times \mathbb{R}^{m \times d} \times \mathbb{R}^{n} \times \mathbb{R}^{n \times l} \rightarrow \mathbb{R}^{n}, \\
G & : \Omega \times[0, T] \times \mathbb{R}^{n} \times \mathbb{R}^{m} \times \mathbb{R}^{n \times l} \times \mathbb{R}^{m \times d} \times \mathbb{R}^{m} \times \mathbb{R}^{m \times d} \rightarrow \mathbb{R}^{m \times l}, \\
g & : \Omega \times[0, T] \times \mathbb{R}^{n} \times \mathbb{R}^{m} \times \mathbb{R}^{n \times l} \times \mathbb{R}^{m \times d} \times \mathbb{R}^{n} \times \mathbb{R}^{n \times l} \rightarrow \mathbb{R}^{n \times d}, \\
\Phi & : \Omega \times \mathbb{R}^{n} \rightarrow \mathbb{R}^{m} .
\end{array}
$$

Given an $m \times n$ full-rank matrix $H$. Let us introduce some notation:

$$
u=\left(\begin{array}{c}
y \\
p \\
z \\
q
\end{array}\right), \quad\left(\begin{array}{c}
y_{\delta}(\cdot) \\
p_{\delta+}(\cdot) \\
z_{\delta}(\cdot) \\
q_{\delta+}(\cdot)
\end{array}\right)=\left(\begin{array}{c}
\alpha \\
\mu \\
\beta \\
v
\end{array}\right), A(t, u, \alpha, \mu, \beta, v)=\left(\begin{array}{c}
-H^{\top} F(t, u, \mu, v) \\
H f(t, u, \alpha, \beta) \\
-H^{\top} G(t, u, \mu, v) \\
H g(t, u, \alpha, \beta)
\end{array}\right) .
$$

where $H^{\top} G=\left(H^{\top} G_{1} \cdots H^{\top} G_{l}\right)$ and $H g=\left(H g_{1} \cdots H g_{d}\right)$.

Similar to $[23,35,47]$, we present the definition of solution to FBDSDEs (5) as follows:

Definition 1. A quadruple of $\mathcal{F}_{t}$-measurable stochastic processes $(y, p, z, q): \Omega \times[-\delta, T] \times$ $[0, T+\delta] \times[-\delta, T] \times[0, T+\delta] \rightarrow \mathbb{R}^{n} \times \mathbb{R}^{m} \times \mathbb{R}^{n \times l} \times \mathbb{R}^{m \times d}$ is called a solution of FBDSDE (5), if $(y, p, z, q) \in L_{\mathcal{F}}^{2}\left(-\delta, T ; \mathbb{R}^{n}\right) \times L_{\mathcal{F}}^{2}\left(0, T+\delta ; \mathbb{R}^{m}\right) \times L_{\mathcal{F}}^{2}\left(-\delta, T ; \mathbb{R}^{n \times l}\right) \times L_{\mathcal{F}}^{2}\left(0, T+\delta ; \mathbb{R}^{m \times d}\right)$ and satisfies FBDSDE (5).

We suppose the following Assumption (H3) holds:

\section{Hypothesis 3 (H3).}

(i) $\quad \int_{0}^{T}\langle A(t, u, \alpha, \mu, \beta, v)-A(t, \bar{u}, \bar{\alpha}, \bar{\mu}, \bar{\beta}, \bar{v}), u-\bar{u}\rangle \mathrm{d} t$

$\leq \int_{0}^{T}\left[-\mu_{1}\left(|H(y-\bar{y})|^{2}+|H(z-\bar{z})|^{2}\right)-\mu_{2}\left(\left|H^{\top}(p-\bar{p})\right|^{2}+\left|H^{\top}(q-\bar{q})\right|^{2}\right)\right] \mathrm{d} t$,

$\forall u=(y, p, z, q), \bar{u}=(\bar{y}, \bar{p}, \bar{z}, \bar{q}) \in \mathbb{R}^{n} \times \mathbb{R}^{m} \times \mathbb{R}^{n \times l} \times \mathbb{R}^{m \times d} ;$

(ii) $\langle\Phi(y)-\Phi(\bar{y}), H(y-\bar{y})\rangle \geq \beta_{1}|H(y-\bar{y})|^{2}, \forall y, \bar{y} \in \mathbb{R}^{n}$;

where $\mu_{1}, \mu_{2}$ and $\beta_{1}$ are given non-negative constants with $\mu_{1}+\mu_{2}>0$, and $\mu_{2}+\beta_{1}>0$. Moreover we have $\mu_{1}>0, \beta_{1}>0$ (resp., $\left.\mu_{2}>0\right)$ when $m>n$ $($ resp., $m<n)$; 
(iii) for each $u, \alpha, \mu, \beta, v, A(\cdot, u, \alpha, \mu, \beta, v)$ is an $\mathcal{F}_{t}$-measurable vector process defined on $[0, T]$ with $A(\cdot, 0) \in L_{\mathcal{F}}^{2}(0, T)$, and for each $y \in \mathbb{R}^{n}, \Phi(y)$ is an $\mathcal{F}_{T}$-measurable random vector with $\Phi(0) \in L^{2}\left(\mathcal{F}_{T} ; \mathbb{R}^{m}\right)$;

(iv) $A(t, u, \alpha, \mu, \beta, v)$ and $\Phi$ satisfy the Lipschitz conditions: there exist constants $k>0$ and $0<\lambda<1$ such that $\forall u, \bar{u}, \alpha, \bar{\alpha}, \mu, \bar{\mu}, \beta, \bar{\beta}, v, \bar{v}, \forall t \in[0, T]$,

$|f(t, u, \alpha, \beta)-f(t, \bar{u}, \bar{\alpha}, \bar{\beta})| \leq k(|u-\bar{u}|+|\alpha-\bar{\alpha}|+|\beta-\bar{\beta}|)$,

$|F(t, u, \mu, v)-F(t, \bar{u}, \bar{\mu}, \bar{v})| \leq k\left(|u-\bar{u}|+\mathbb{E}^{\mathcal{F}_{t}}[|\mu-\bar{\mu}|+|v-\bar{v}|]\right)$,

$|g(t, u, \alpha, \beta)-g(t, \bar{u}, \bar{\alpha}, \bar{\beta})|$

$\leq k(|y-\bar{y}|+|p-\bar{p}|+|q-\bar{q}|+|\alpha-\bar{\alpha}|)+\lambda(|z-\bar{z}|+|\beta-\bar{\beta}|)$,

$|G(t, u, \mu, v)-G(t, \bar{u}, \bar{\mu}, \bar{v})|$

$\leq k\left(|y-\bar{y}|+|p-\bar{p}|+|z-\bar{z}|+\mathbb{E}^{\mathcal{F}_{t}}[|\mu-\bar{\mu}|]\right)+\lambda\left(|q-\bar{q}|+\mathbb{E}^{\mathcal{F}_{t}}[|v-\bar{v}|]\right)$,

$|\Phi(y)-\Phi(\bar{y})| \leq k|y-\bar{y}|$.

By the similar method of $[23,35,47]$, we can prove the following Theorem 1 . For the convenience of the reader, we present the skeleton of the proof in Section 6.

Theorem 1. Under assumption (H3), then FBDSDE (5) has a unique solution $(y, p, z, q) \in$ $L_{\mathcal{F}}^{2}\left(-\delta, T ; \mathbb{R}^{n}\right) \times L_{\mathcal{F}}^{2}\left(0, T+\delta ; \mathbb{R}^{m}\right) \times L_{\mathcal{F}}^{2}\left(-\delta, T ; \mathbb{R}^{n \times l}\right) \times L_{\mathcal{F}}^{2}\left(0, T+\delta ; \mathbb{R}^{m \times d}\right)$.

\section{Necessary Maximum Principle}

For convex admissible control sets, the classical method to obtain the necessary optimality condition is the convex perturbation method. Let $u(\cdot)=\left(u_{1}(\cdot), u_{2}(\cdot)\right)$ be an equilibrium point of Problem (A) and $(y(\cdot), z(\cdot))$ be the corresponding optimal trajectory. Let $\left(v_{1}(\cdot), v_{2}(\cdot)\right)$ be such that $\left(u_{1}(\cdot)+v_{1}(\cdot), u_{2}(\cdot)+v_{2}(\cdot)\right) \in \mathcal{U}_{1} \times \mathcal{U}_{2}$. Since $\mathcal{U}_{1}$ and $\mathcal{U}_{2}$ are convex, for any $0 \leq \rho \leq 1,\left(u_{1}^{\rho}(\cdot), u_{2}^{\rho}(\cdot)\right)=\left(u_{1}(\cdot)+\rho v_{1}(\cdot), u_{1}(\cdot)+\rho v_{1}(\cdot)\right)$ is also in $\mathcal{U}_{1} \times \mathcal{U}_{2}$. As illustrated before, we denote by $\left(y^{u_{1}^{\rho}}(\cdot), z^{\left.u_{1}^{\rho}(\cdot)\right)}\right.$ and $\left(y^{u_{2}^{\rho}}(\cdot), z^{u_{2}^{\rho}}(\cdot)\right)$ the corresponding state trajectories of the game system in Equation (3) along with the controls $\left(u_{1}^{\rho}(\cdot), u_{2}(\cdot)\right)$ and $\left(u_{1}(\cdot), u_{2}^{\rho}(\cdot)\right)$.

For convenience, we use the following notations throughout this paper:

$$
\begin{aligned}
\varphi(t) & =\varphi\left(t, y(t), z(t), y_{\delta}(t), z_{\delta}(t), u_{1}(t), u_{2}(t)\right), \\
\varphi^{u_{1}^{\rho}}(t) & =\varphi\left(t, y^{\rho}(t), z^{\rho}(t), y_{\delta}^{\rho}(t), z_{\delta}^{\rho}(t), u_{1}^{\rho}(t), u_{2}(t)\right), \\
\varphi^{u_{2}^{\rho}}(t) & =\varphi\left(t, y^{\rho}(t), z^{\rho}(t), y_{\delta}^{\rho}(t), z_{\delta}^{\rho}(t), u_{1}(t), u_{2}^{\rho}(t)\right),
\end{aligned}
$$

where $\varphi$ means one of $f, g, l_{i}, i=1,2$.

We bring in the following variational equation:

$$
\left\{\begin{aligned}
\mathrm{d} y_{i}^{1}(t)= & {\left[f_{y}(t) y_{i}^{1}(t)+f_{z}(t) z_{i}^{1}(t)+f_{y_{\delta}}(t) y_{i \delta}^{1}(t)+f_{z_{\delta}}(t) z_{i \delta}^{1}(t)+f_{v_{i}}(t) v_{i}(t)\right] \mathrm{d} t } \\
& +\left[g_{y}(t) y_{i}^{1}(t)+g_{z}(t) z_{i}^{1}(t)+g_{y_{\delta}}(t) y_{i \delta}^{1}(t)+g_{z_{\delta}}(t) z_{i \delta}^{1}(t)+g_{v_{i}}(t) v_{i}(t)\right] \overrightarrow{\mathrm{d}} W(t) \\
& -z_{i}^{1}(t) \stackrel{\grave{\mathrm{d}}}{B}(t) \\
y_{i}^{1}(t)= & 0, z_{i}^{1}(t)=0, t \in[-\delta, 0],(i=1,2) .
\end{aligned}\right.
$$

By (H1) and Theorem 3.1.1 in [20], it is easy to see that there is a unique adapted solution to Equation (6).

For $t \in[0, T], \rho>0$, we set

$$
\begin{aligned}
& \tilde{y}_{i}^{\rho}(t)=\frac{y^{u_{i}^{\rho}}(t)-y(t)}{\rho}-y_{i}^{1}(t), \\
& \tilde{z}_{i}^{\rho}(t)=\frac{z^{u_{i}^{\rho}}(t)-z(t)}{\rho}-z_{i}^{1}(t),(i=1,2) .
\end{aligned}
$$

We have the following: 
Lemma 1. Let the hypotheses (H1) and (H2) be true. Then, for $i=1,2$,

$$
\begin{aligned}
& \lim _{\rho \rightarrow 0} \sup _{0 \leq t \leq T} \mathbb{E}\left|\tilde{y}_{i}^{\rho}(t)\right|^{2}=0, \\
& \lim _{\rho \rightarrow 0} \mathbb{E} \int_{0}^{T}\left|\tilde{z}_{i}^{\rho}(t)\right|^{2} \mathrm{~d} t=0 .
\end{aligned}
$$

Proof of Lemma 1. For $i=1$, we have

$$
\left\{\begin{aligned}
\mathrm{d} \tilde{y}_{1}^{\rho}(t)= & {\left[\frac{1}{\rho}\left(f^{u_{1}^{\rho}}(t)-f(t)\right)-f_{y}(t) y_{1}^{1}(t)-f_{z}(t) z_{1}^{1}(t)-f_{y_{\delta}}(t) y_{1 \delta}^{1}(t)\right.} \\
& \left.-f_{z_{\delta}}(t) z_{1 \delta}^{1}(t)-f_{v_{1}}(t) v_{1}(t)\right] \mathrm{d} t \\
& +\left[\frac{1}{\rho}\left(g^{u_{1}^{\rho}}(t)-g(t)\right)-g_{y}(t) y_{1}^{1}(t)-g_{z}(t) z_{1}^{1}(t)-g_{y_{\delta}}(t) y_{1 \delta}^{1}(t)\right. \\
& \left.-g_{z_{\delta}}(t) z_{1 \delta}^{1}(t)-g_{v_{1}}(t) v_{1}(t)\right] \overrightarrow{\mathrm{d}} W(t)-\tilde{z}_{1}^{\rho}(t) \overleftarrow{\mathrm{d}} B(t), \\
\tilde{y}_{1}^{\rho}(t)= & 0, \tilde{z}_{1}^{\rho}(t)=0, t \in[-\delta, 0],
\end{aligned}\right.
$$

or

$$
\left\{\begin{aligned}
\mathrm{d} \tilde{y}_{1}^{\rho}(t)= & {\left[A_{1}^{\rho}(t, \cdot) \tilde{y}_{1}^{\rho}(t)+\bar{A}_{1}^{\rho}(t, \cdot) \tilde{y}_{1 \delta}^{\rho}(t)+B_{1}^{\rho}(t, \cdot) \tilde{z}_{1}^{\rho}(t)\right.} \\
& \left.+\bar{B}_{1}^{\rho}(t, \cdot) \tilde{z}_{1 \delta}^{\rho}(t)+G_{1}^{\rho}(t)\right] \mathrm{d} t \\
& +\left[C_{1}^{\rho}(t, \cdot) \tilde{y}_{1}^{\rho}(t)+\bar{C}_{1}^{\rho}(t, \cdot) \tilde{y}_{1 \delta}^{\rho}(t)+D_{1}^{\rho}(t, \cdot) \tilde{z}_{1}^{\rho}(t)\right. \\
& \left.+\bar{D}_{1}^{\rho}(t, \cdot) \tilde{z}_{1 \delta}^{\rho}(t)+G_{2}^{\rho}(t)\right] \overrightarrow{\mathrm{d}} W(t)-\tilde{z}_{1}^{\rho}(t) \stackrel{\mathrm{d}}{B}(t), \\
\tilde{y}_{1}^{\rho}(t)= & 0, \tilde{z}_{1}^{\rho}(t)=0, t \in[-\delta, 0],
\end{aligned}\right.
$$

where we denote

$$
\begin{aligned}
& A_{1}^{\rho}(t)=\int_{0}^{1} f_{y}\left(t, y_{1}(t)+\lambda\left(y^{u_{1}^{\rho}}(t)-y(t)\right), z^{u_{1}^{\rho}}(t), y_{\delta}^{u_{1}^{\rho}}(t), z_{\delta}^{u_{1}^{\rho}}(t), u_{1}^{\rho}(t), u_{2}(t)\right) \mathrm{d} \lambda \\
& B_{1}^{\rho}(t)=\int_{0}^{1} f_{z}\left(t, y(t), z_{1}(t)+\lambda\left(z^{u_{1}^{\rho}}(t)-z(t)\right), y_{\delta}^{u_{1}^{\rho}}(t), z_{\delta}^{u_{1}^{\rho}}(t), u_{1}^{\rho}(t), u_{2}(t)\right) \mathrm{d} \lambda, \\
& \bar{A}_{1}^{\rho}(t)=\int_{0}^{1} f_{y_{\delta}}\left(t, y(t), z(t), y_{1 \delta}(t)+\lambda \rho\left(y_{1 \delta}^{1}(t)+\tilde{y}_{1 \delta}^{\rho}(t)\right), z_{\delta}^{u_{1}^{\rho}}(t), u_{1}^{\rho}(t), u_{2}(t)\right) \mathrm{d} \lambda, \\
& \bar{B}_{1}^{\rho}(t)=\int_{0}^{1} f_{z_{\delta}}\left(t, y(t), z(t), y_{\delta}(t), z_{1 \delta}(t)+\lambda \rho\left(z_{1 \delta}^{1}(t)+\tilde{z}_{1 \delta}^{\rho}(t)\right), u_{1}^{\rho}(t), u_{2}(t)\right) \mathrm{d} \lambda, \\
& C_{1}^{\rho}(t)=\int_{0}^{1} g_{y}\left(t, y_{1}(t)+\lambda\left(y^{u_{1}^{\rho}}(t)-y(t)\right), z^{u_{1}^{\rho}}(t), y_{\delta}^{u_{1}^{\rho}}(t), z_{\delta}^{u_{1}^{\rho}}(t), u_{1}^{\rho}(t), u_{2}(t)\right) \mathrm{d} \lambda, \\
& D_{1}^{\rho}(t)=\int_{0}^{1} g_{z}\left(t, y(t), z_{1}(t)+\lambda\left(z^{u_{1}^{\rho}}(t)-z(t)\right), y_{\delta}^{u_{1}^{\rho}}(t), z_{\delta}^{u_{1}^{\rho}}(t), u_{1}^{\rho}(t), u_{2}(t)\right) \mathrm{d} \lambda, \\
& \bar{C}_{1}^{\rho}(t)=\int_{0}^{1} g_{y_{\delta}}\left(t, y(t), z(t), y_{1 \delta}(t)+\lambda \rho\left(y_{1 \delta}^{1}(t)+\tilde{y}_{1 \delta}^{\rho}(t)\right), z_{\delta}^{u_{1}^{\rho}}(t), u_{1}^{\rho}(t), u_{2}(t)\right) \mathrm{d} \lambda, \\
& \bar{D}_{1}^{\rho}(t)=\int_{0}^{1} g_{z_{\delta}}\left(t, y(t), z(t), y_{\delta}(t), z_{1 \delta}(t)+\lambda \rho\left(z_{1 \delta}^{1}(t)+\tilde{z}_{1 \delta}^{\rho}(t)\right), u_{1}^{\rho}(t), u_{2}(t)\right) \mathrm{d} \lambda, \\
& G_{1}^{\rho}(t)=\int_{0}^{1}\left(f_{v_{1}}\left(t, y(t), z(t), y_{\delta}(t), z_{\delta}(t), u_{1}(t)+\rho \lambda v_{1}(t), u_{2}(t)\right)-f_{v_{1}}(t)\right) v_{1}(t) \mathrm{d} \lambda \\
& +\left[A_{1}^{\rho}(t)-f_{y}(t)\right] y_{1}^{1}(t)+\left[B_{1}^{\rho}(t)-f_{z}(t)\right] z_{1}^{1}(t) \\
& +\left[\bar{A}_{1}^{\rho}(t)-f_{y_{\delta}}(t)\right] y_{1 \delta}^{1}(t)+\left[\bar{B}_{1}^{\rho}(t)-f_{z_{\delta}}(t)\right] z_{1 \delta}^{1}(t), \\
& G_{2}^{\rho}(t)=\int_{0}^{1}\left(g_{v_{1}}\left(t, y(t), z(t), y_{\delta}(t), z_{\delta}(t), u_{1}(t)+\rho \lambda v_{1}(t), u_{2}(t)\right)-g_{v_{1}}(t)\right) v_{1}(t) \mathrm{d} \lambda \\
& +\left[C_{1}^{\rho}(t)-g_{y}(t)\right] y_{1}^{1}(t)+\left[D_{1}^{\rho}(t)-g_{z}(t)\right] z_{1}^{1}(t) \\
& +\left[\bar{C}_{1}^{\rho}(t)-g_{y_{\delta}}(t)\right] y_{1 \delta}^{1}(t)+\left[\bar{D}_{1}^{\rho}(t)-g_{z_{\delta}}(t)\right] z_{1 \delta}^{1}(t) \text {. }
\end{aligned}
$$


Using Itô's formula to $\left|\tilde{y}_{1}^{\rho}(t)\right|^{2}$ on $[0, t]$, through $(\mathrm{H} 1)$, we get

$$
\begin{aligned}
& \mathbb{E}\left|\tilde{y}_{1}^{\rho}(t)\right|^{2}+\mathbb{E} \int_{0}^{t}\left|\tilde{z}_{1}^{\rho}(s)\right|^{2} \mathrm{~d} s \\
\leq & C_{0} \mathbb{E} \int_{0}^{t}\left|\tilde{y}_{1}^{\rho}(s)\right|^{2} \mathrm{~d} s+\frac{1}{2} \mathbb{E} \int_{0}^{t}\left|\tilde{z}_{1}^{\rho}(s)\right|^{2} \mathrm{~d} s+C_{1}\left(\mathbb{E} \int_{0}^{t}\left(\left|G_{1}^{\rho}(s)\right|^{2}+\left|G_{2}^{\rho}(s)\right|^{2}\right) \mathrm{d} s .\right.
\end{aligned}
$$

Applying Grownwall's inequalities, we can easily get the desired result. Again, we can prove that for $i=2$. The proof is complete.

Based on $\left(u_{1}(\cdot), u_{2}(\cdot)\right)$ being an equilibrium point of Problem $(\mathrm{A})$, then

$$
\begin{aligned}
& \rho^{-1}\left[J_{1}\left(u_{1}^{\rho}(\cdot), u_{2}(\cdot)\right)-J_{1}\left(u_{1}(\cdot), u_{2}(\cdot)\right)\right] \geq 0, \\
& \rho^{-1}\left[J_{2}\left(u_{1}(\cdot), u_{2}^{\rho}(\cdot)\right)-J_{2}\left(u_{1}(\cdot), u_{2}(\cdot)\right)\right] \geq 0 .
\end{aligned}
$$

From Equations (9) and (10) and Lemma 1, we obtain the following variational inequality.

Lemma 2. Let assumptions (H1) and (H2) hold. Then

$$
\begin{aligned}
& \mathbb{E} \int_{0}^{T}\left[l_{i y}(t) y_{i}^{1}(t)+l_{i z}(t) z_{i}^{1}(t)+l_{i y_{\delta}}(t) y_{i \delta}^{1}(t)+l_{i z_{\delta}}(t) z_{i \delta}^{1}(t)+l_{i v_{i}}(t) v_{i}(t)\right] \mathrm{d} t \\
& +\mathbb{E}\left[\Phi_{i y}(y(T)) y_{i}^{1}(T)\right] \geq 0,(i=1,2) .
\end{aligned}
$$

Proof of Lemma 2. For $i=1$, from Equation (7), we derive

$$
\begin{aligned}
& \rho^{-1} \mathbb{E}\left[\Phi_{1}\left(y^{u_{1}^{\rho}}(T)\right)-\Phi_{1}(y(T))\right] \\
= & \rho^{-1} \mathbb{E} \int_{0}^{1} \Phi_{1 y}\left(y(T)+\lambda\left(y^{u_{1}^{\rho}}(T)-y(T)\right)\right)\left(y^{u_{1}^{\rho}}(T)-y(T)\right) \mathrm{d} \lambda \\
\rightarrow & \mathbb{E}\left[\Phi_{1 y}(y(T)) y_{1}^{1}(T)\right], \rho \rightarrow 0 .
\end{aligned}
$$

Similarly, we have

$$
\begin{aligned}
& \rho^{-1}\left\{\mathbb{E} \int_{0}^{T}\left[l_{1}^{u_{1}^{\rho}}(t)-l_{1}(t)\right] \mathrm{d} t\right\} \\
\rightarrow & \mathbb{E} \int_{0}^{T}\left[l_{1 y}(t) y_{1}^{1}(t)+l_{1 z}(t) z_{1}^{1}(t)+l_{1 y_{\delta}}(t) y_{1 \delta}^{1}(t)+l_{1 z_{\delta}}(t) z_{1 \delta}^{1}(t)+l_{1 v_{1}}(t) v_{1}(t)\right] \mathrm{d} t, \rho \rightarrow 0 .
\end{aligned}
$$

Let $\rho \rightarrow 0$ in Equation (9), so, for $i=1$, Equation (11) is established. Similarly, we can prove that the conclusion holds for $i=2$. The proof is complete.

Let us define the Hamiltonian function $H_{i}:[0, T] \times \mathbb{R}^{n} \times \mathbb{R}^{n \times d} \times \mathbb{R}^{n} \times \mathbb{R}^{n \times d} \times \mathbb{R}^{k_{1}} \times$ $\mathbb{R}^{k_{2}} \times \mathbb{R}^{n} \times \mathbb{R}^{n \times l} \rightarrow \mathbb{R}, i=1,2$ as follows:

$$
\begin{aligned}
& H_{i}\left(t, y, z, y_{\delta}, z_{\delta}, v_{1}, v_{2}, p_{i}, q_{i}\right) \\
= & \left\langle f\left(t, y, z, y_{\delta}, z_{\delta}, v_{1}, v_{2}\right), p_{i}\right\rangle+\left\langle g\left(t, y, z, y_{\delta}, z_{\delta}, v_{1}, v_{2}\right), q_{i}\right\rangle+l_{i}\left(t, y, z, y_{\delta}, z_{\delta}, v_{1}, v_{2}\right), i=1,2 .
\end{aligned}
$$

We introduce the following adjoint equation

$$
\left\{\begin{aligned}
-\mathrm{d} p_{i}(t)= & \left\{H_{i y}\left(t, \Theta(t), u_{1}(t), u_{2}(t), p_{i}(t), q_{i}(t)\right)\right. \\
& \left.+\mathbb{E}^{\mathcal{F}_{t}}\left[H_{i y_{\delta}}\left(t+\delta, \Theta(t+\delta), u_{1}(t+\delta), u_{2}(t+\delta), p_{i}(t+\delta), q_{i}(t+\delta)\right)\right]\right\} \mathrm{d} t \\
& +\left\{H_{i z}\left(t, \Theta(t), u_{1}(t), u_{2}(t), p_{i}(t), q_{i}(t)\right)\right. \\
& \left.+\mathbb{E}^{\mathcal{F}_{t}}\left[H_{i z_{\delta}}\left(t+\delta, \Theta(t+\delta), u_{1}(t+\delta), u_{2}(t+\delta), p_{i}(t+\delta), q_{i}(t+\delta)\right)\right]\right\} \overleftarrow{\mathrm{d}} B(t) \\
& -q_{i}(t) \overrightarrow{\mathrm{d}} W(t), \\
p_{i}(T)= & \Phi_{i y}(y(T)), p_{i}(t)=0, q_{i}(t)=0, t \in[T, T+\delta],(i=1,2) .
\end{aligned}\right.
$$

where $\Theta(t):=\left(y(t), z(t), y_{\delta}(t), z_{\delta}(t)\right)$. 
Remark 1. It is easy to see that the adjoint Equation (12) above is a linear anticipated BDSDE, then the unique solvability of Equation (12) can be guaranteed by theorem 3.2 in [45] and theorem 2.4 in [46].

Theorem 2 (Necessary maximum principle). Suppose $(H 1)$ and $(H 2)$ hold, and $\left(u_{1}(\cdot), u_{2}(\cdot)\right)$ is an equilibrium point of Problem $(A)$ and $(y(\cdot), z(\cdot))$ is the corresponding state trajectory. Then we have

$$
\begin{aligned}
& \left\langle H_{1 v_{1}}\left(t, \Theta(t), u_{1}(t), u_{2}(t), p_{1}(t), q_{1}(t)\right), v_{1}-u_{1}(t)\right\rangle \geq 0, \\
& \left\langle H_{2 v_{2}}\left(t, \Theta(t), u_{1}(t), u_{2}(t), p_{2}(t), q_{2}(t)\right), v_{2}-u_{2}(t)\right\rangle \geq 0,
\end{aligned}
$$

hold for any $\left(v_{1}, v_{2}\right) \in U_{1} \times U_{2}$, a.e., a.s., where $\left(p_{i}(\cdot), q_{i}(\cdot)(i=1,2)\right.$ is the solution of the adjoint Equation (12).

Proof of Theorem 2. For $i=1$. Using Itô's formula to $\left\langle p_{1}(t), y_{1}^{1}(t)\right\rangle$, we obtain

$$
\begin{aligned}
& \mathbb{E}\left\langle\Phi_{1 y}(y(T)), y_{1}^{1}(T)\right\rangle \\
&= \mathbb{E} \int_{0}^{T}\left\langle p_{1}(t), f_{y}(t) y_{1}^{1}(t)+f_{z}(t) z_{1}^{1}(t)+f_{y_{\delta}}(t) y_{1 \delta}^{1}(t)+f_{z_{\delta}}(t) z_{1 \delta}^{1}(t)+f_{v_{1}}(t) v_{1}(t)\right\rangle \mathrm{d} t \\
&+ \mathbb{E} \int_{0}^{T}\left\langle-f_{y}^{\top}(t) p_{1}(t)-g_{y}^{\top}(t) q_{1}(t)-l_{1 y}(t)\right. \\
&\left.\quad+\mathbb{E}^{\mathcal{F}_{t}}\left[-f_{y_{\delta}}^{\top}(t+\delta) p_{1}(t+\delta)-g_{y_{\delta}}^{\top}(t+\delta) q_{1}(t+\delta)-l_{1 y_{\delta}}(t+\delta)\right], y_{1}^{1}(t)\right\rangle \mathrm{d} t \\
&+\mathbb{E} \int_{0}^{T}\left\langle q_{1}(t), g_{y}(t) y_{1}^{1}(t)+g_{z}(t) z_{1}^{1}(t)+g_{y_{\delta}}(t) y_{1 \delta}^{1}(t)+g_{z_{\delta}}(t) z_{1 \delta}^{1}(t)+g_{v_{1}}(t) v_{1}(t)\right\rangle \mathrm{d} t \\
&+\mathbb{E} \int_{0}^{T}\left\langle-f_{z}^{\top}(t) p_{1}(t)-g_{z}^{\top}(t) q_{1}(t)-l_{1 z}(t)\right. \\
&\left.\quad+\mathbb{E}^{\mathcal{F}_{t}}\left[-f_{z_{\delta}}^{T}(t+\delta) p_{1}(t+\delta)-g_{z_{\delta}}^{T}(t+\delta) q_{1}(t+\delta)-l_{1 z_{\delta}}(t+\delta)\right], z_{1}^{1}(t)\right\rangle \mathrm{d} t .
\end{aligned}
$$

Combining the initial conditions and the termination conditions, we get

$$
\begin{aligned}
& \mathbb{E} \int_{0}^{T}\left[\left\langle p_{1}(t), f_{y_{\delta}}(t) y_{1 \delta}^{1}(t)\right\rangle-\left\langle\mathbb{E}^{\mathcal{F}_{t}}\left[f_{y_{\delta}}^{\top}(t+\delta) p_{1}(t+\delta)\right], y_{1}^{1}(t)\right\rangle\right] \mathrm{d} t \\
= & \mathbb{E} \int_{0}^{T}\left\langle p_{1}(t), f_{y_{\delta}}(t) y_{1 \delta}^{1}(t)\right\rangle \mathrm{d} t-\mathbb{E} \int_{\delta}^{T+\delta}\left\langle f_{y_{\delta}}^{\top}(t) p_{1}(t), y_{1 \delta}^{1}(t)\right\rangle \mathrm{d} t \\
= & \mathbb{E} \int_{0}^{\delta}\left\langle p_{1}(t), f_{y_{\delta}}(t) y_{1 \delta}^{1}(t)\right\rangle \mathrm{d} t-\mathbb{E} \int_{T}^{T+\delta}\left\langle f_{y_{\delta}}^{\top}(t) p_{1}(t), y_{1 \delta}^{1}(t)\right\rangle \mathrm{d} t \\
= & 0 .
\end{aligned}
$$

Similarly, we have

$$
\begin{aligned}
& \mathbb{E} \int_{0}^{T}\left[\left\langle p_{1}(t), f_{z_{\delta}}(t) z_{1 \delta}^{1}(t)\right\rangle-\left\langle\mathbb{E}^{\mathcal{F}_{t}}\left[f_{z_{\delta}}^{\top}(t+\delta) p_{1}(t+\delta)\right], z_{1}^{1}(t)\right\rangle\right] \mathrm{d} t=0, \\
& \mathbb{E} \int_{0}^{T}\left[\left\langle q_{1}(t), g_{y_{\delta}}(t) y_{1 \delta}^{1}(t)\right\rangle-\left\langle\mathbb{E}^{\mathcal{F}_{t}}\left[g_{y_{\delta}}^{\top}(t+\delta) q_{1}(t+\delta)\right], y_{1}^{1}(t)\right\rangle\right] \mathrm{d} t=0, \\
& \mathbb{E} \int_{0}^{T}\left[\left\langle q_{1}(t), g_{z_{\delta}}(t) z_{1 \delta}^{1}(t)\right\rangle-\left\langle\mathbb{E}^{\mathcal{F}_{t}}\left[g_{z_{\delta}}^{\top}(t+\delta) q_{1}(t+\delta)\right], z_{1}^{1}(t)\right\rangle\right] \mathrm{d} t=0 .
\end{aligned}
$$

Then, we get

$$
\begin{aligned}
& \mathbb{E} \int_{0}^{T}\left[l_{1 y}(t) y_{1}^{1}(t)+l_{1 z}(t) z_{1}^{1}(t)+l_{1 y_{\delta}}(t) y_{1 \delta}^{1}(t)+l_{1 z_{\delta}}(t) z_{1 \delta}^{1}(t)+l_{1 v_{1}}(t) v_{1}(t)\right] \mathrm{d} t \\
& +\mathbb{E}\left\langle\Phi_{1 y}(y(T)), y_{1}^{1}(T)\right\rangle \\
= & \mathbb{E} \int_{0}^{T}\left\langle f_{v_{1}}^{\top}(t) p_{1}(t)+g_{v_{1}}^{\top}(t) q_{1}(t)+l_{1 v_{1}}(t), v_{1}(t)\right\rangle \mathrm{d} t .
\end{aligned}
$$

According to Lemma 2, we have

$$
\mathbb{E} \int_{0}^{T}\left\langle H_{1 v_{1}}\left(t, \Theta(t), u_{1}(t), u_{2}(t), p_{1}(t), q_{1}(t)\right), v_{1}(t)\right\rangle \mathrm{d} t \geq 0 .
$$


Because $v_{1}(t)$ satisfies $u_{1}(t)+v_{1}(t) \in \mathcal{U}_{1}$, we have

$$
\mathbb{E} \int_{0}^{T}\left\langle H_{1 v_{1}}\left(t, \Theta(t), u_{1}(t), u_{2}(t), p_{1}(t), q_{1}(t)\right), v_{1}-u_{1}(t)\right\rangle \mathrm{d} t \geq 0, \forall v_{1} \in U_{1},
$$

which means that

$$
\mathbb{E}\left\langle H_{1 v_{1}}\left(t, \Theta(t), u_{1}(t), u_{2}(t), p_{1}(t), q_{1}(t)\right), v_{1}-u_{1}(t)\right\rangle \geq 0, \text { a.s. }
$$

At present, take an arbitrary element $F$ of $\sigma$-algebra $\mathcal{F}_{t}$, and set

$$
w(t)=v \mathbf{1}_{F}+u(t) \mathbf{1}_{\Omega-F} .
$$

Obviously, $w(t)$ is an admissible control.

We apply the inequality in Equation (13) to $w(t)$, and get

$$
\mathbb{E}\left[\mathbf{1}_{F}\left\langle H_{1 v_{1}}\left(t, \Theta(t), u_{1}(t), u_{2}(t), p_{1}(t), q_{1}(t)\right), v_{1}-u_{1}(t)\right\rangle\right] \geq 0, \forall F \in \mathcal{F}_{t},
$$

which contains that

$$
\mathbb{E}\left[\left\langle H_{1 v_{1}}\left(t, \Theta(t), u_{1}(t), u_{2}(t), p_{1}(t), q_{1}(t)\right), v_{1}-u_{1}(t)\right\rangle \mid \mathcal{F}_{t}\right] \geq 0 .
$$

The expression within the conditional expectation is $\mathcal{F}_{t}$-measurable, so the result follows. Following the above proof, we can prove that the other inequality is true for any $v_{2} \in U_{2}$. The proof is completed.

\section{Sufficient Maximum Principle}

In this section, the sufficient maximum principle for Problem (A) is investigated. Let $(y(t), z(t)$, $\left.u_{1}(t), u_{2}(t)\right)$ be a quintuple satisfying Equation (3), and suppose there exists a solution $\left(p_{i}(t), q_{i}(t)\right)$ of the corresponding adjoint forward SDE (12). We assume that:

Hypothesis 4 (H4). For $i=1,2$, for all $t \in[0, T], H_{i}\left(t, y, z, y_{\delta}, z_{\delta}, v_{1}, v_{2}, p_{i}, q_{i}\right)$ is convex in $\left(y, z, y_{\delta}, z_{\delta}\right.$, $\left.v_{1}, v_{2}\right)$, and $\Phi_{i}(y)$ is convex in $y$.

Theorem 3 (Sufficient maximum principle). Suppose (H1), (H2) and (H4) are true. In addition, the following conditions hold

$$
\begin{aligned}
& H_{1}\left(t, \Theta(t), u_{1}(t), u_{2}(t), p_{1}(t), q_{1}(t)\right)=\min _{v_{1} \in \mathcal{U}_{1}} H_{1}\left(t, \Theta(t), v_{1}(t), u_{2}(t), p_{1}(t), q_{1}(t)\right), \\
& H_{2}\left(t, \Theta(t), u_{1}(t), u_{2}(t), p_{2}(t), q_{2}(t)\right)=\min _{v_{2} \in \mathcal{U}_{2}} H_{2}\left(t, \Theta(t), u_{1}(t), v_{2}(t), p_{2}(t), q_{2}(t)\right) .
\end{aligned}
$$

Then $\left(u_{1}(\cdot), u_{2}(\cdot)\right)$ is an equilibrium point of Problem $(A)$.

Proof of Theorem 3. For any $v_{1}(\cdot) \in \mathcal{U}_{1}$, we consider

$$
\begin{aligned}
& J_{1}\left(v_{1}(\cdot), u_{2}(\cdot)\right)-J_{1}\left(u_{1}(\cdot), u_{2}(\cdot)\right) \\
= & \mathbb{E} \int_{0}^{T}\left[l_{1}\left(t, \Theta(t), v_{1}(t), u_{2}(t)\right)-l_{1}\left(t, \Theta(t), u_{1}(t), u_{2}(t)\right)\right] \mathrm{d} t \\
+ & \mathbb{E}\left[\Phi_{1}\left(y^{v_{1}}(T)\right)-\Phi_{1}(y(T))\right] .
\end{aligned}
$$


Now we put into use Itô's formula to $\left\langle p_{1}(t), y^{v_{1}}(t)-y(t)\right\rangle$ on $[0, T]$, and get

$$
\begin{aligned}
& \mathbb{E}\left\langle\Phi_{1 y}(y(T)), y^{v_{1}}(T)-y(T)\right\rangle \\
= & -\mathbb{E} \int_{0}^{T}\left\langle y^{v_{1}}(t)-y(t), H_{1 y}\left(t, \Theta(t), u_{1}(t), u_{2}(t), p_{1}(t), q_{1}(t)\right)\right. \\
& \left.\quad+\mathbb{E}^{\mathcal{F}_{t}}\left[H_{1 y_{\delta}}\left(t+\delta, \Theta(t+\delta), u_{1}(t+\delta), u_{2}(t+\delta), p_{1}(t+\delta), q_{1}(t+\delta)\right)\right]\right\rangle \mathrm{d} t \\
+ & \mathbb{E} \int_{0}^{T}\left\langle p_{1}(t), f\left(t, \Theta(t), v_{1}(t), u_{2}(t)\right)-f\left(t, \Theta(t), u_{1}(t), u_{2}(t)\right)\right\rangle \mathrm{d} t \\
- & \mathbb{E} \int_{0}^{T}\left\langle z^{v_{1}}(t)-z(t), H_{1 z}\left(t, \Theta(t), u_{1}(t), u_{2}(t), p_{1}(t), q_{1}(t)\right)\right. \\
& \left.+\mathbb{E}^{\mathcal{F}_{t}}\left[H_{1 z_{\delta}}\left(t+\delta, \Theta(t+\delta), u_{1}(t+\delta), u_{2}(t+\delta), p_{1}(t+\delta), q_{1}(t+\delta)\right)\right]\right\rangle \mathrm{d} t \\
+ & \mathbb{E} \int_{0}^{T}\left\langle q_{1}(t), g\left(t, \Theta(t), v_{1}(t), u_{2}(t)\right)-g\left(t, \Theta(t), u_{1}(t), u_{2}(t)\right)\right\rangle \mathrm{d} t .
\end{aligned}
$$

Since $\Phi_{1}$ is convex, we have

$$
\Phi_{1}\left(y^{v_{1}}(T)\right)-\Phi_{1}(y(T)) \geq\left\langle\Phi_{1 y}(y(T)), y^{v_{1}}(T)-y(T)\right\rangle .
$$

Then, we have

$$
\begin{aligned}
& J_{1}\left(v_{1}(\cdot), u_{2}(\cdot)\right)-J_{1}\left(u_{1}(\cdot), u_{2}(\cdot)\right) \\
& \geq \quad \mathbb{E} \int_{0}^{T}\left[H_{1}\left(t, \Theta(t), v_{1}(t), u_{2}(t), p_{1}(t), q_{1}(t)\right)-H_{1}\left(t, \Theta(t), u_{1}(t), u_{2}(t), p_{1}(t), q_{1}(t)\right)\right] \mathrm{d} t \\
&-\mathbb{E} \int_{0}^{T}\left\langle y^{v_{1}}(t)-y(t), H_{1 y}\left(t, \Theta(t), u_{1}(t), u_{2}(t), p_{1}(t), q_{1}(t)\right)\right. \\
&\left.\quad+\mathbb{E}^{\mathcal{F}_{t}}\left[H_{1 y_{\delta}}\left(t+\delta, \Theta(t+\delta), u_{1}(t+\delta), u_{2}(t+\delta), p_{1}(t+\delta), q_{1}(t+\delta)\right)\right]\right\rangle \mathrm{d} t \\
&-\mathbb{E} \int_{0}^{T}\left\langle z^{v_{1}}(t)-z(t), H_{1 z}\left(t, \Theta(t), u_{1}(t), u_{2}(t), p_{1}(t), q_{1}(t)\right)\right. \\
&\left.\quad+\mathbb{E}^{\mathcal{F}_{t}}\left[H_{1 z_{\delta}}\left(t+\delta, \Theta(t+\delta), u_{1}(t+\delta), u_{2}(t+\delta), p_{1}(t+\delta), q_{1}(t+\delta)\right)\right]\right\rangle \mathrm{d} t .
\end{aligned}
$$

Based on the convexity of $H_{1}$ with respect to $\left(y, z, y_{\delta}, z_{\delta}, v_{1}, v_{2}\right)$, we achieve

$$
\begin{aligned}
& H_{1}\left(t, \Theta(t), v_{1}(t), u_{2}(t), p_{1}(t), q_{1}(t)\right)-H_{1}\left(t, \Theta(t), u_{1}(t), u_{2}(t), p_{1}(t), q_{1}(t)\right) \\
\geq & \left\langle y^{v_{1}}(t)-y(t), H_{1 y}\left(t, \Theta(t), u_{1}(t), u_{2}(t), p_{1}(t), q_{1}(t)\right)\right\rangle \\
& +\left\langle z^{v_{1}}(t)-z(t), H_{1 z}\left(t, \Theta(t), u_{1}(t), u_{2}(t), p_{1}(t), q_{1}(t)\right)\right\rangle \\
& +\left\langle y_{\delta}^{v_{1}}(t)-y_{\delta}(t), H_{1 y_{\delta}}\left(t, \Theta(t), u_{1}(t), u_{2}(t), p_{1}(t), q_{1}(t)\right)\right\rangle \\
& +\left\langle z_{\delta}^{v_{1}}(t)-z_{\delta}(t), H_{1 z_{\delta}}\left(t, \Theta(t), u_{1}(t), u_{2}(t), p_{1}(t), q_{1}(t)\right)\right\rangle \\
& +\left\langle v_{1}(t)-u_{1}(t), H_{1 v_{1}}\left(t, \Theta(t), u_{1}(t), u_{2}(t), p_{1}(t), q_{1}(t)\right)\right\rangle .
\end{aligned}
$$

Noticing the fact that

$$
\begin{aligned}
& \mathbb{E} \int_{0}^{T}\left\langle y_{\delta}^{v_{1}}(t)-y_{\delta}(t), H_{1 y_{\delta}}\left(t, \Theta(t), u_{1}(t), u_{2}(t), p_{1}(t), q_{1}(t)\right)\right\rangle \mathrm{d} t \\
& -\mathbb{E} \int_{0}^{T}\left\langle y^{v_{1}}(t)-y(t), \mathbb{E}^{\mathcal{F}_{t}}\left[H_{1 y_{\delta}}\left(t+\delta, \Theta(t+\delta), u_{1}(t+\delta), u_{2}(t+\delta), p_{1}(t+\delta), q_{1}(t+\delta)\right)\right]\right\rangle \mathrm{d} t \\
= & \mathbb{E} \int_{0}^{T}\left\langle y_{\delta}^{v_{1}}(t)-y_{\delta}(t), H_{1 y_{\delta}}\left(t, \Theta(t), u_{1}(t), u_{2}(t), p_{1}(t), q_{1}(t)\right)\right\rangle \mathrm{d} t \\
& -\mathbb{E} \int_{\delta}^{T+\delta}\left\langle y_{\delta}^{v_{1}}(t)-y_{\delta}(t), H_{1 y_{\delta}}\left(t, \Theta(t), u_{1}(t), u_{2}(t), p_{1}(t), q_{1}(t)\right)\right\rangle \mathrm{d} t \\
= & \mathbb{E} \int_{0}^{\delta}\left\langle y_{\delta}^{v_{1}}(t)-y_{\delta}(t), H_{1 y_{\delta}}\left(t, \Theta(t), u_{1}(t), u_{2}(t), p_{1}(t), q_{1}(t)\right)\right\rangle \mathrm{d} t \\
& -\mathbb{E} \int_{T}^{T+\delta}\left\langle y_{\delta}^{v_{1}}(t)-y_{\delta}(t), H_{1 y_{\delta}}\left(t, \Theta(t), u_{1}(t), u_{2}(t), p_{1}(t), q_{1}(t)\right)\right\rangle \mathrm{d} t \\
= & 0 .
\end{aligned}
$$


Similarly, we have

$$
\begin{aligned}
& \mathbb{E} \int_{0}^{T}\left\langle z_{\delta}^{v_{1}}(t)-z_{\delta}(t), H_{1 z_{\delta}}\left(t, \Theta(t), u_{1}(t), u_{2}(t), p_{1}(t), q_{1}(t)\right)\right\rangle \mathrm{d} t \\
& -\mathbb{E} \int_{0}^{T}\left\langle z^{v_{1}}(t)-z(t), \mathbb{E}^{\mathcal{F}_{t}}\left[H_{1 z_{\delta}}\left(t+\delta, \Theta(t+\delta), u_{1}(t+\delta), u_{2}(t+\delta), p_{1}(t+\delta), q_{1}(t+\delta)\right)\right]\right\rangle \mathrm{d} t \\
= & 0 .
\end{aligned}
$$

Then, we get

$$
\begin{aligned}
& J_{1}\left(v_{1}(\cdot), u_{2}(\cdot)\right)-J_{1}\left(u_{1}(\cdot), u_{2}(\cdot)\right) \\
\geq & \mathbb{E} \int_{0}^{T}\left\langle H_{1 v_{1}}\left(t, \Theta(t), u_{1}(t), u_{2}(t), p_{1}(t), q_{1}(t)\right), v_{1}(t)-u_{1}(t)\right\rangle \mathrm{d} t .
\end{aligned}
$$

Finally, by the necessary optimality conditions in Equation (14), we obtain

$$
J_{1}\left(v_{1}(\cdot), u_{2}(\cdot)\right)-J_{1}\left(u_{1}(\cdot), u_{2}(\cdot)\right) \geq 0 .
$$

This implies that

$$
J_{1}\left(u_{1}(\cdot), u_{2}(\cdot)\right)=\min _{v_{1}(\cdot) \in \mathcal{U}_{1}} J_{1}\left(v_{1}(\cdot), u_{2}(\cdot)\right)
$$

In the same way

$$
J_{2}\left(u_{1}(\cdot), u_{2}(\cdot)\right)=\min _{v_{2}(\cdot) \in \mathcal{U}_{2}} J_{2}\left(u_{1}(\cdot), v_{2}(\cdot)\right) .
$$

Hence, the desired conclusion is drawn. The proof is completed.

\section{Applications in LQ Doubly Stochastic Games with Delay}

In this section, our maximal principle is used for the nonzero sum differential game problem of LQ doubly stochastic systems with delay. To simplify the notation, let us assume that $d=l=1$. The control system is

$$
\left\{\begin{aligned}
\mathrm{d} y(t)= & {\left[A(t) y(t)+B(t) z(t)+\bar{A}(t) y_{\delta}(t)+\bar{B}(t) z_{\delta}(t)+E_{1}(t) v_{1}(t)\right.} \\
& \left.+E_{2}(t) v_{2}(t)\right] \mathrm{d} t-z(t) \overleftarrow{\mathrm{d}} B(t) \\
& +\left[C(t) y(t)+D(t) z(t)+\bar{C}(t) y_{\delta}(t)+\bar{D}(t) z_{\delta}(t)\right] \overrightarrow{\mathrm{d}} W(t), t \in[0, T] \\
y(t)= & \xi(t), t \in[-\delta, 0] \\
z(t)= & \eta(t), t \in[-\delta, 0]
\end{aligned}\right.
$$

where $(\xi(\cdot), \eta(\cdot)) \in L_{\mathcal{F}}^{2}\left(-\delta, T ; \mathbb{R}^{n}\right)$ is the initial path of $(y, z) . A, \bar{A}, B, \bar{B}, C, \bar{C}, D, \bar{D}$ are $n \times n$ bounded matrices, $v_{1}(t)$ and $v_{2}(t), t \in[0, T]$ are two admissible control processes, i.e., $\mathcal{F}_{t}$-measurable squareintegrable processes taking values in $\mathbb{R}^{k} . E_{1}$ and $E_{2}$ are $n \times k$ bounded matrices. We denote $J_{1}\left(v_{1}(\cdot), v_{2}(\cdot)\right)$ and $J_{2}\left(v_{1}(\cdot), v_{2}(\cdot)\right)$, which are the cost functionals corresponding to the players 1 and 2:

$$
\begin{aligned}
J_{i}\left(v_{1}(\cdot), v_{2}(\cdot)\right)= & \frac{1}{2} \mathbb{E}\left\{\int_{0}^{T}\left[\left\langle M_{i}(t) y(t), y(t)\right\rangle+\left\langle R_{i}(t) z(t), z(t)\right\rangle+\left\langle N_{i}(t) v_{i}(t), v_{i}(t)\right\rangle\right] \mathrm{d} t\right. \\
& \left.+\left\langle Q_{i} y(T), y(T)\right\rangle\right\}, i=1,2,
\end{aligned}
$$

where $M_{i}(t), R_{i}(t), Q_{i}, i=1,2$ are $n \times n$ non-negative symmetric bounded matrices, and $N_{i}(t), i=1,2$ are $k \times k$ positive symmetric bounded matrices and the inverse $N_{i}^{-1}(t), i=1,2$ are also bounded. Our task is to find $\left(u_{1}(\cdot), u_{2}(\cdot)\right) \in \mathbb{R}^{k} \times \mathbb{R}^{k}$ such that

$$
\left\{\begin{array}{l}
J_{1}\left(u_{1}(\cdot), u_{2}(\cdot)\right)=\min _{v_{1}(\cdot) \in \mathcal{U}_{1}} J_{1}\left(v_{1}(\cdot), u_{2}(\cdot)\right), \\
J_{2}\left(u_{1}(\cdot), u_{2}(\cdot)\right)=\min _{v_{2}(\cdot) \in \mathcal{U}_{2}} J_{2}\left(u_{1}(\cdot), v_{2}(\cdot)\right) .
\end{array}\right.
$$

We need the following assumption:

Hypothesis 5 (H5). $E_{i}\left(N_{i}\right)^{-1} E_{i}^{\top} S=S E_{i}\left(N_{i}\right)^{-1} E_{i}^{\top}$ 
where $S=A, \bar{A}, B, \bar{B}, C, \bar{C}, D, \bar{D}$, and $i=1,2$. Now, with the help of the above general FBDSDE, the explicit expression for the Nash equilibrium point of the above game problem can be obtained.

Theorem 4. The mapping

$$
\left(u_{1}(t), u_{2}(t)\right)=\left(N_{1}^{-1}(t) E_{1}^{\top}(t) p_{1}(t), N_{2}^{-1}(t) E_{2}^{\top}(t) p_{2}(t)\right), t \in[0, T]
$$

is one Nash equilibrium point for the above game problems in Equations (16)-(18), where $\left(y(t), z(t), p_{1}(t), p_{2}(t), q_{1}(t), q_{2}(t)\right)$ is the solution of the following general FBDSDE:

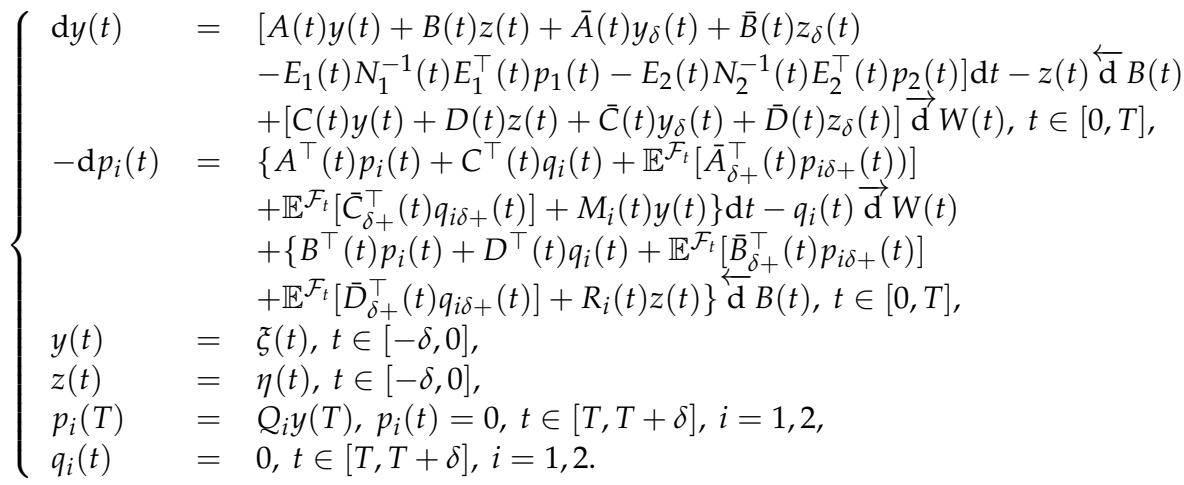

Similar to [31,48], the proof of Theorem 4 is easy to give, and we have therefore excluded it.

For sake of clarity, we give the following Problem (S), which is a special case of Problem (A). To simplify the notation, let us assume that $n=d=l=k=1$. The control system is

$$
\left\{\begin{array}{l}
\mathrm{d} y(t)=\left[y_{\delta}(t)+z_{\delta}(t)+v_{1}(t)+v_{2}(t)\right] \mathrm{d} t-z(t) \overleftarrow{\mathrm{d}} B(t)+y_{\delta}(t) \overrightarrow{\mathrm{d}} W(t), t \in[0, T] \\
y(t)=\xi(t), t \in[-\delta, 0] \\
z(t)=\eta(t), t \in[-\delta, 0]
\end{array}\right.
$$

where $(\xi(\cdot), \eta(\cdot)) \in L_{\mathcal{F}}^{2}(-\delta, T ; \mathbb{R})$ is the initial path of $(y, z) . v_{1}(t)$ and $v_{2}(t), t \in[0, T]$ are two admissible control processes, i.e., $\mathcal{F}_{t}$-measurable square-integrable processes taking values in $\mathbb{R}$. We denote $J_{1}\left(v_{1}(\cdot), v_{2}(\cdot)\right)$ and $J_{2}\left(v_{1}(\cdot), v_{2}(\cdot)\right)$, which are the cost functionals corresponding to the players 1 and 2:

$$
J_{i}\left(v_{1}(\cdot), v_{2}(\cdot)\right)=\mathbb{E}\left\{\frac{1}{2} \int_{0}^{T} v_{i}^{2}(t) \mathrm{d} t+y(T)\right\}, i=1,2 .
$$

Our task is to find $\left(u_{1}(\cdot), u_{2}(\cdot)\right) \in \mathbb{R}^{k} \times \mathbb{R}^{k}$ such that

$$
\left\{\begin{array}{l}
J_{1}\left(u_{1}(\cdot), u_{2}(\cdot)\right)=\min _{v_{1}(\cdot) \in \mathcal{U}_{1}} J_{1}\left(v_{1}(\cdot), u_{2}(\cdot)\right), \\
J_{2}\left(u_{1}(\cdot), u_{2}(\cdot)\right)=\min _{v_{2}(\cdot) \in \mathcal{U}_{2}} J_{2}\left(u_{1}(\cdot), v_{2}(\cdot)\right) .
\end{array}\right.
$$

Then the Hamiltonian functions are

$$
H_{i}\left(t, y_{\delta}, z_{\delta}, v_{1}, v_{2}, p_{i}, q_{i}\right)=\left[y_{\delta}+z_{\delta}+v_{1}+v_{2}\right] p_{i}+y_{\delta} q_{i}+\frac{1}{2}, i=1,2,
$$

where $\left(p_{1}(t), p_{2}(t), q_{1}(t), q_{2}(t)\right)$ is the solution of the following adjoint equations:

$$
\left\{\begin{aligned}
-\mathrm{d} p_{i}(t)= & \left.\left\{\mathbb{E}^{\mathcal{F}_{t}}\left[p_{i \delta+}(t)\right)\right]+\mathbb{E}^{\mathcal{F}_{t}}\left[q_{i \delta+}(t)\right]\right\} \mathrm{d} t-q_{i}(t) \overrightarrow{\mathrm{d}} W(t) \\
& +\mathbb{E}^{\mathcal{F}_{t}}\left[p_{i \delta+}(t)\right] \mathrm{d} B(t), t \in[0, T] \\
p_{i}(T)= & 1, p_{i}(t)=0, t \in[T, T+\delta], i=1,2 \\
q_{i}(t)= & 0, t \in[T, T+\delta], i=1,2
\end{aligned}\right.
$$

It is easy to see that the above equation is the anticipated BDSDE, which is solvable theorem 3.2 in [45] and theorem 2.4 in [46]. From the maximum principle, we get that $\left(u_{1}(t), u_{2}(t)\right)=$ $\left(p_{1}(t), p_{2}(t)\right), t \in[0, T]$ is one Nash equilibrium point for the above game in Equations (16)-(18). 


\section{The Proof of Theorem 1}

Proof of Theorem 1. Since the initial path of $(y, z)$ in $[-\delta, 0]$ and the terminal conditions and trajectories of $(p, q)$ in $[T, T+\delta]$ are given in advance, we only need to consider $\left(y_{t}, p_{t}, z_{t}, q_{t}\right), 0 \leq t \leq T$.

Uniqueness Let $U=(y, p, z, q)$ and $\bar{U}=(\bar{y}, \bar{p}, \bar{z}, \bar{q})$ be two solutions of Equation (3). We set $\widehat{U}=U-\bar{U}=(\widehat{y}, \widehat{p}, \widehat{z}, \widehat{q})=(y-\bar{y}, p-\bar{p}, z-\bar{z}, q-\bar{q})$.

Applying Itô's formula to $\langle H \widehat{y}, \widehat{p}\rangle$ on $[0, T]$, we have

$$
\begin{aligned}
& \mathbb{E}\langle H \widehat{y}(T), \Phi(y(T))-\Phi(\bar{y}(T))\rangle \\
= & \mathbb{E} \int_{0}^{T}\langle A(t, U(t), \alpha(t), \mu(t), \beta(t), v(t))-A(t, \bar{U}(t), \bar{\alpha}(t), \bar{\mu}(t), \bar{\beta}(t), \bar{v}(t)), \widehat{U}(t)\rangle \mathrm{d} t \\
\leq & -\mu_{1} \mathbb{E} \int_{0}^{T}\left(|H(y(t)-\bar{y}(t))|^{2}+|H(z(t)-\bar{z}(t))|^{2}\right) \mathrm{d} t \\
& -\mu_{2} \mathbb{E} \int_{0}^{T}\left(\left|H^{\top}(p(t)-\bar{p}(t))\right|^{2}+\left|H^{\top}(q(t)-\bar{q}(t))\right|^{2}\right) \mathrm{d} t .
\end{aligned}
$$

By virtue of (H3), it follows that

$$
\begin{aligned}
& \mu_{1} \mathbb{E} \int_{0}^{T}\left(|H(y(t)-\bar{y}(t))|^{2}+|H(z(t)-\bar{z}(t))|^{2}\right) \mathrm{d} t \\
& +\mu_{2} \mathbb{E} \int_{0}^{T}\left(\left|H^{\top}(p(t)-\bar{p}(t))\right|^{2}+\left|H^{\top}(q(t)-\bar{q}(t))\right|^{2}\right) \mathrm{d} t \leq 0 .
\end{aligned}
$$

If $m>n, \mu_{1}>0$, then we have $|H(y(t)-\bar{y}(t))|^{2} \equiv 0$ and $|H(z(t)-\bar{z}(t))|^{2} \equiv 0$. Thus $y(t) \equiv \bar{y}(t)$ and $z(t) \equiv \bar{z}(t)$. In particular, $\Phi(y(T))=\Phi(\bar{y}(T))$. Consequently, from the uniqueness result of the anticipated BDSDE (see $[45,46])$, it follows that $p(t) \equiv \bar{p}(t)$ and $q(t) \equiv \bar{q}(t)$.

If $m<n, \mu_{2}>0$, then we have $\left|H^{\top}(p(t)-\bar{p}(t))\right|^{2} \equiv 0$ and $\left|H^{\top}(q(t)-\bar{q}(t))\right|^{2} \equiv 0$. Thus $p(t) \equiv \bar{p}(t)$ and $q(t) \equiv \bar{q}(t)$. From the uniqueness result of the delayed doubly SDE (see [20]), it follows that $y(t) \equiv \bar{y}(t)$ and $z(t) \equiv \bar{z}(t)$.

Similarly to the above arguments, the desired result can be obtained easily in the case $n=m$. The uniqueness is proved.

The proof of the existence is a combination of the above technique and a priori estimate technique introduced by Peng [49]. We divide the proof of existence into three cases: $m>n, m<n$ and $m=n$.

Case 1 If $m>n$, then $\mu_{1}>0, \beta_{1}>0$. We consider the following family of FBDSDEs parametrized by $\alpha \in[0,1]$

$$
\left\{\begin{aligned}
\mathrm{d} y(t)= & {\left[\alpha f\left(t, U(t), y_{\delta}(t), z_{\delta}(t)\right)+f_{0}(t)\right] \mathrm{d} t-z(t) \overleftarrow{\mathrm{d}} B(t) } \\
& +\left[\alpha g\left(t, U(t), y_{\delta}(t), z_{\delta}(t)\right)+g_{0}(t)\right] \overrightarrow{\mathrm{d}} W(t), t \in[0, T], \\
-\mathrm{d} p(t)= & {\left[\alpha F\left(t, U(t), p_{\delta+}(t), q_{\delta+}(t)\right)+(1-\alpha) \mu_{1} H y(t)+F_{0}(t)\right] \mathrm{d} t } \\
& +\left[\alpha G\left(t, U(t), p_{\delta+}(t), q_{\delta+}(t)\right)+(1-\alpha) \mu_{1} H z(t)+G_{0}(t)\right] \overleftarrow{\mathrm{d}} B(t) \\
& -q(t) \overrightarrow{\mathrm{d}} W(t), t \in[0, T], \\
y(t)= & \phi(t), t \in[-\delta, 0], \\
z(t)= & \psi(t), t \in[-\delta, 0], \\
p(T)= & \alpha \Phi(y(T))+(1-\alpha) H y(T)+\varphi, \\
p(t)= & \xi(t), t \in[T, T+\delta], \\
q(t)= & \eta(t), t \in[T, T+\delta],
\end{aligned}\right.
$$

where $U=(y, p, z, q)$ and $\left(F_{0}, f_{0}, G_{0}, g_{0}\right) \in L_{\mathcal{F}}^{2}\left(0, T ; \mathbb{R}^{m+n+m \times l+n \times d}\right)$ and $\varphi \in L^{2}\left(\mathcal{F}_{T} ; \mathbb{R}^{m}\right)$ are arbitrarily given vector-valued random variables. When $\alpha=1$ the existence of the solution of Equation (21) implies clearly that of Equation (5). Due to the existence and uniqueness of the delayed doubly SDE (see [20]), when $\alpha=0$, the Equation (21) is uniquely solvable. The following a priori lemma is a key step in the proof of the method of continuation. It shows that for a fixed $\alpha=\alpha_{0} \in[0,1)$, if Equation (21) is uniquely solvable, then it is also uniquely solvable for any $\alpha \in\left[\alpha_{0}, \alpha_{0}+\gamma_{0}\right]$, for some positive constant $\gamma_{0}$ independent of $\alpha_{0}$.

Lemma 3. We assume $m>n$. Under assumptions (H3), there exists a positive constant $\gamma_{0}$ such that if a priori, for each $\varphi \in L^{2}\left(\mathcal{F}_{T} ; \mathbb{R}^{m}\right),\left(F_{0}, f_{0}, G_{0}, g_{0}\right) \in L_{\mathcal{F}}^{2}\left(0, T ; \mathbb{R}^{m+n+m \times l+n \times d}\right)$, Equation (16) is uniquely 
solvable for some $\alpha_{0} \in[0,1)$, then for each $\alpha \in\left[\alpha_{0}, \alpha_{0}+\gamma_{0}\right]$, and $\varphi \in L^{2}\left(\mathcal{F}_{T} ; \mathbb{R}^{m}\right),\left(F_{0}, f_{0}, G_{0}, g_{0}\right) \in$ $L_{\mathcal{F}}^{2}\left(0, T ; \mathbb{R}^{m+n+m \times l+n \times d}\right)$, Equation (16) is also uniquely solvable in $L_{\mathcal{F}}^{2}\left(-\delta, T ; \mathbb{R}^{n}\right) \times L_{\mathcal{F}}^{2}\left(0, T+\delta ; \mathbb{R}^{m}\right) \times$ $L_{\mathcal{F}}^{2}\left(-\delta, T ; \mathbb{R}^{n \times l}\right) \times L_{\mathcal{F}}^{2}\left(0, T+\delta ; \mathbb{R}^{m \times d}\right)$.

Proof of Lemma 3. Since for $\varphi \in L^{2}\left(\mathcal{F}_{T} ; \mathbb{R}^{m}\right),\left(F_{0}, f_{0}, G_{0}, g_{0}\right) \in L_{\mathcal{F}}^{2}\left(0, T ; \mathbb{R}^{m+n+m \times l+n \times d}\right)$, there exists a unique solution to Equation (16) for $\alpha=\alpha_{0}$. Thus for each $\bar{U}=(\bar{y}, \bar{p}, \bar{z}, \bar{q}) \in L_{\mathcal{F}}^{2}\left(-\delta, T ; \mathbb{R}^{n}\right) \times$ $L_{\mathcal{F}}^{2}\left(0, T+\delta ; \mathbb{R}^{m}\right) \times L_{\mathcal{F}}^{2}\left(-\delta, T ; \mathbb{R}^{n \times l}\right) \times L_{\mathcal{F}}^{2}\left(0, T+\delta ; \mathbb{R}^{m \times d}\right)$, there exists a unique quadruple $U=$ $(y, p, z, q) \in L_{\mathcal{F}}^{2}\left(-\delta, T ; \mathbb{R}^{n}\right) \times L_{\mathcal{F}}^{2}\left(0, T+\delta ; \mathbb{R}^{m}\right) \times L_{\mathcal{F}}^{2}\left(-\delta, T ; \mathbb{R}^{n \times l}\right) \times L_{\mathcal{F}}^{2}\left(0, T+\delta ; \mathbb{R}^{m \times d}\right)$ satisfying the following equations

$$
\left\{\begin{aligned}
& \mathrm{d} y(t)= {\left[\alpha_{0} f\left(t, U(t), y_{\delta}(t), z_{\delta}(t)\right)+\gamma f\left(t, \bar{U}(t), \bar{y}_{\delta}(t), \bar{z}_{\delta}(t)\right)+f_{0}(t)\right] \mathrm{d} t } \\
&+\left[\alpha_{0} g\left(t, \vec{U}(t), y_{\delta}(t), z_{\delta}(t)\right)+\gamma g\left(t, \bar{U}(t), \bar{y}_{\delta}(t), \bar{z}_{\delta}(t)\right)\right. \\
&\left.+g_{0}(t)\right] \overrightarrow{\mathrm{d}} W(t)-z(t) \overleftarrow{\mathrm{d}} B(t), t \in[0, T], \\
&-\mathrm{d} p(t)= {\left[\alpha_{0} F\left(t, U(t), p_{\delta+}(t), q_{\delta+}(t)\right)+\left(1-\alpha_{0}\right) \mu_{1} H y(t)\right.} \\
&\left.+\gamma\left(F\left(t, \bar{U}(t), \bar{p}_{\delta+}(t), \bar{q}_{\delta+}(t)\right)-\mu_{1} H \bar{y}(t)\right)+F_{0}(t)\right] \mathrm{d} t \\
&+\left[\alpha_{0} G\left(t, U(t), p_{\delta+}(t), q_{\delta+}(t)\right)+\left(1-\alpha_{0}\right) \mu_{1} H z(t)\right. \\
&\left.+\gamma\left(G\left(t, \bar{U}(t), \bar{p}_{\delta+}(t), \bar{q}_{\delta+}(t)\right)-\mu_{1} H \bar{y}(t)\right)+G_{0}(t)\right] \overleftarrow{\mathrm{d}} B(t) \\
&-q(t) \overrightarrow{\mathrm{d}} W(t), t \in[0, T], \\
&= \phi(t), t \in[-\delta, 0], \\
& y(t)= \psi(t), t \in[-\delta, 0], \\
& z(t)= \alpha_{0} \Phi(y(T))+\left(1-\alpha_{0}\right) H y(T)+\gamma(\Phi(\bar{y}(T))-H \bar{y}(T))+\varphi, \\
& p(T)= \xi(t), t \in[T, T+\delta], \\
& p(t)= \eta(t), t \in[T, T+\delta], \\
& q(t)=
\end{aligned}\right.
$$

where $\gamma \in(0,1)$ is independent of $\alpha_{0}$. We will prove that the mapping defined by

$$
\begin{aligned}
& U=I_{\alpha_{0}+\gamma}(\bar{U}): \\
& L_{\mathcal{F}}^{2}\left(-\delta, T ; \mathbb{R}^{n}\right) \times L_{\mathcal{F}}^{2}\left(0, T+\delta ; \mathbb{R}^{m}\right) \times L_{\mathcal{F}}^{2}\left(-\delta, T ; \mathbb{R}^{n \times l}\right) \times L_{\mathcal{F}}^{2}\left(0, T+\delta ; \mathbb{R}^{m \times d}\right) \\
& \rightarrow L_{\mathcal{F}}^{2}\left(-\delta, T ; \mathbb{R}^{n}\right) \times L_{\mathcal{F}}^{2}\left(0, T+\delta ; \mathbb{R}^{m}\right) \times L_{\mathcal{F}}^{2}\left(-\delta, T ; \mathbb{R}^{n \times l}\right) \times L_{\mathcal{F}}^{2}\left(0, T+\delta ; \mathbb{R}^{m \times d}\right)
\end{aligned}
$$

is contractive for a small enough $\gamma>0$.

Let $\bar{U}^{\prime}=\left(\bar{y}^{\prime}, \bar{p}^{\prime}, \bar{z}^{\prime}, \bar{q}^{\prime}\right) \in L_{\mathcal{F}}^{2}\left(-\delta, T ; \mathbb{R}^{n}\right) \times L_{\mathcal{F}}^{2}\left(0, T+\delta ; \mathbb{R}^{m}\right) \times L_{\mathcal{F}}^{2}\left(-\delta, T ; \mathbb{R}^{n \times l}\right) \times L_{\mathcal{F}}^{2}(0, T+\delta ;$ $\left.\mathbb{R}^{m \times d}\right)$ and $\left(y^{\prime}, p^{\prime}, z^{\prime}, q^{\prime}\right)=U^{\prime}=I_{\alpha_{0}+\delta}\left(\bar{U}^{\prime}\right)$.

$$
\begin{aligned}
& \widehat{\bar{U}}=\bar{U}-\bar{U}^{\prime}=(\widehat{\bar{y}}, \hat{\bar{p}}, \widehat{\bar{z}}, \widehat{\bar{q}})=\left(\bar{y}-\bar{y}^{\prime}, \bar{p}-\bar{p}^{\prime}, \bar{z}-\bar{z}^{\prime}, \bar{q}-\bar{q}^{\prime}\right), \\
& \widehat{U}=U-U^{\prime}=(\widehat{y}, \widehat{p}, \widehat{z}, \widehat{q})=\left(y-y^{\prime}, p-p^{\prime}, z-z^{\prime}, q-q^{\prime}\right) .
\end{aligned}
$$

Applying Itô's formula to $\langle H \widehat{y}, \widehat{p}\rangle$ on $[0, T]$, it follows that

$$
\begin{aligned}
& \left(1-\alpha_{0}+\alpha_{0} \beta_{1}\right) \mathbb{E}|H \widehat{y}(T)|^{2}+\mu_{1} \mathbb{E} \int_{0}^{T}\left(|H \widehat{y}(t)|^{2}+|H \widehat{z}(t)|^{2}\right) \mathrm{d} t \\
\leq & \gamma C \mathbb{E} \int_{0}^{T}\left(|\widehat{\hat{U}}(t)|^{2}+\left|\widehat{\bar{y}}_{\delta}(t)\right|^{2}+\left|\widehat{\bar{z}}_{\delta}(t)\right|^{2}+\left|\widehat{\bar{p}}_{\delta+}(t)\right|^{2}+\left|\widehat{\bar{q}}_{\delta+}(t)\right|^{2}\right) \mathrm{d} t \\
& +\gamma C \mathbb{E} \int_{0}^{T}|\widehat{U}(t)|^{2} \mathrm{~d} t+\gamma C\left(\mathbb{E}|\widehat{y}(T)|^{2}+\mathbb{E}|\widehat{\bar{y}}(T)|^{2}\right) \\
\leq & \gamma C\left[\mathbb{E} \int_{0}^{T}\left(|\widehat{\bar{y}}(t)|^{2}+|\widehat{\bar{z}}(t)|^{2}\right) \mathrm{d} t+\mathbb{E} \int_{0}^{T+\delta}\left(|\widehat{\bar{p}}(t)|^{2}+|\widehat{\bar{q}}(t)|^{2}\right) \mathrm{d} t\right] \\
& +\gamma C \mathbb{E} \int_{0}^{T}|\widehat{U}(t)|^{2} \mathrm{~d} t+\gamma C\left(\mathbb{E}|\widehat{y}(T)|^{2}+\mathbb{E}|\widehat{\bar{y}}(T)|^{2}\right),
\end{aligned}
$$

with some constant $C>0$. Hereafter, $C$ will be some generic constant, which can be different from line to line and depends only on the Lipschitz constants $k, \lambda, \mu_{1}, \beta_{1}, H$ and $T$. It is obvious that $1-\alpha_{0}+\alpha_{0} \beta_{1} \geq \beta, \beta=\min \left(1, \beta_{1}\right)>0$.

On the other hand, for the difference of the solutions $(\widehat{p}, \widehat{q})=\left(p-p^{\prime}, q-q^{\prime}\right)$, we apply a standard method of estimation. Applying Itô's formula to $|\widehat{p}(t)|^{2}$ on $[t, T]$, we have 


$$
\begin{aligned}
& \mathbb{E} \int_{0}^{T}\left(|\widehat{p}(t)|^{2}+|\widehat{q}(t)|^{2}\right) \mathrm{d} t \\
\leq & \gamma C\left[\mathbb{E} \int_{0}^{T}\left(|\widehat{\bar{y}}(t)|^{2}+|\widehat{\bar{z}}(t)|^{2}\right) \mathrm{d} t+\mathbb{E} \int_{0}^{T+\delta}\left(|\widehat{\bar{p}}(t)|^{2}+|\widehat{\bar{q}}(t)|^{2}\right) \mathrm{d} t\right] \\
& +C\left(\mathbb{E}|\widehat{y}(T)|^{2}+\delta \mathbb{E}|\widehat{\bar{y}}(T)|^{2}\right)+C \mathbb{E} \int_{0}^{T}\left(|\widehat{y}(t)|^{2}+|\widehat{z}(t)|^{2}\right) \mathrm{d} t .
\end{aligned}
$$
have

Combining the estimates in Equations (22) and (23), for a sufficiently large constant $C>0$, we

$$
\begin{aligned}
& \mathbb{E} \int_{-\delta}^{T}\left(|\widehat{y}(t)|^{2}+|\widehat{z}(t)|^{2}\right) \mathrm{d} t+\mathbb{E} \int_{0}^{T+\delta}\left(|\widehat{p}(t)|^{2}+|\widehat{q}(t)|^{2}\right) \mathrm{d} t+\mathbb{E}|\widehat{y}(T)|^{2} \\
\leq & \gamma C \mathbb{E} \int_{-\delta}^{T}\left(|\widehat{\bar{y}}(t)|^{2}+|\widehat{\bar{z}}(t)|^{2}\right) \mathrm{d} t+\mathbb{E} \int_{0}^{T+\delta}\left(|\widehat{\bar{p}}(t)|^{2}+|\widehat{\bar{q}}(t)|^{2}\right) \mathrm{d} t+\mathbb{E}|\widehat{\bar{y}}(T)|^{2} .
\end{aligned}
$$

We now choose $\gamma_{0}=\frac{1}{2 C}$. It is clear that, for each fixed $\gamma \in\left[0, \gamma_{0}\right]$, the mapping $I_{\alpha_{0}+\gamma}$ is contractive in the sense that

$$
\begin{aligned}
& \mathbb{E} \int_{-\delta}^{T}\left(|\widehat{y}(t)|^{2}+|\widehat{z}(t)|^{2}\right) \mathrm{d} t+\mathbb{E} \int_{0}^{T+\delta}\left(|\widehat{p}(t)|^{2}+|\widehat{q}(t)|^{2}\right) \mathrm{d} t+\mathbb{E}|\widehat{y}(T)|^{2} \\
\leq & \frac{1}{2} \mathbb{E} \int_{-\delta}^{T}\left(|\widehat{\bar{y}}(t)|^{2}+|\widehat{\bar{z}}(t)|^{2}\right) \mathrm{d} t+\mathbb{E} \int_{0}^{T+\delta}\left(|\widehat{\bar{p}}(t)|^{2}+|\widehat{\bar{q}}(t)|^{2}\right) \mathrm{d} t+\mathbb{E}|\widehat{\bar{y}}(T)|^{2} .
\end{aligned}
$$

Thus this mapping has a unique fixed point $U=(y, p, z, q) \in L_{\mathcal{F}}^{2}\left(-\delta, T ; \mathbb{R}^{n}\right) \times L_{\mathcal{F}}^{2}\left(0, T+\delta ; \mathbb{R}^{m}\right) \times$ $L_{\mathcal{F}}^{2}\left(-\delta, T ; \mathbb{R}^{n \times l}\right) \times L_{\mathcal{F}}^{2}\left(0, T+\delta ; \mathbb{R}^{m \times d}\right)$, which is the solution of Equation (16) for $\alpha=\alpha_{0}+\gamma$, as $\gamma \in\left[0, \gamma_{0}\right]$. The proof is complete.

Case 2 If $m<n$, then $\mu_{2}>0$. We consider the following equations

$$
\left\{\begin{aligned}
\mathrm{d} y(t)= & {\left[\alpha f\left(t, U(t), y_{\delta}(t), z_{\delta}(t)\right)+(1-\alpha) \mu_{1} H^{\top} p(t)+f_{0}(t)\right] \mathrm{d} t } \\
& +\left[\alpha g\left(t, U(t), y_{\delta}(t), z_{\delta}(t)\right)+(1-\alpha) \mu_{1} H^{\top} q(t)+g_{0}(t)\right] \overrightarrow{\mathrm{d}} W(t) \\
& -z(t) \overleftarrow{\mathrm{d}} B(t), t \in[0, T], \\
-\mathrm{d} p(t)= & {\left[\alpha F\left(t, U(t), p_{\delta+}(t), q_{\delta+}(t)\right)+F_{0}(t)\right] \mathrm{d} t-q(t) \overrightarrow{\mathrm{d}} W(t) } \\
& +\left[\alpha G\left(t, U(t), p_{\delta+}(t), q_{\delta+}(t)\right)+G_{0}(t)\right] \overleftarrow{\mathrm{d}} B(t), t \in[0, T], \\
y(t)= & \phi(t), t \in[-\delta, 0], \\
z(t)= & \psi(t), t \in[-\delta, 0], \\
p(T)= & \alpha \Phi(y(T))+\varphi, \\
p(t)= & \xi(t), t \in[T, T+\delta], \\
q(t)= & \eta(t), t \in[T, T+\delta] .
\end{aligned}\right.
$$

When $\alpha=1$, the existence of the solution of Equation (24) implies clearly that of Equation (16). Due to the existence and uniqueness of the anticipated BDSDE (see $[45,46]$ ), when $\alpha=0$, we know that Equation (24) is uniquely solvable. By the same techniques, we can also prove the following lemma similar to Lemma 3.

Lemma 4. Assume $m<n$. Under assumption (H3), there exists a positive constant $\gamma_{0}$ such that if a priori, for each $\varphi \in L^{2}\left(\mathcal{F}_{T} ; \mathbb{R}^{m}\right)$, and $\left(F_{0}, f_{0}, G_{0}, g_{0}\right) \in L_{\mathcal{F}}^{2}\left(0, T ; \mathbb{R}^{m+n+m \times l+n \times d}\right)$, Equation (24) is uniquely solvable for some $\alpha_{0} \in[0,1)$, then for each $\alpha \in\left[\alpha_{0}, \alpha_{0}+\gamma_{0}\right]$, and $\varphi \in L^{2}\left(\mathcal{F}_{T} ; \mathbb{R}^{m}\right),\left(F_{0}, f_{0}, G_{0}, g_{0}\right) \in$ $L_{\mathcal{F}}^{2}\left(0, T ; \mathbb{R}^{m+n+m \times l+n \times d}\right)$, Equation (24) is also uniquely solvable in $L_{\mathcal{F}}^{2}\left(-\delta, T ; \mathbb{R}^{n}\right) \times L_{\mathcal{F}}^{2}\left(0, T+\delta ; \mathbb{R}^{m}\right) \times$ $L_{\mathcal{F}}^{2}\left(-\delta, T ; \mathbb{R}^{n \times l}\right) \times L_{\mathcal{F}}^{2}\left(0, T+\delta ; \mathbb{R}^{m \times d}\right)$.

Case $3 m=n$. From (H3), we only need to consider two cases as follows:

(1) If $\mu_{1}>0, \mu_{2} \geq 0, \beta_{1}>0$, we can have the same result as Lemma 3.

(2) If $\mu_{1} \geq 0, \mu_{2}>0, \beta_{1} \geq 0$, the same result as Lemma 4 holds.

Now we give the proof of the existence of Theorem 1. 
Proof of the Existence of Theorem 1. For the first case where $m>n$, we know that for each $\varphi \in$ $L^{2}\left(\mathcal{F}_{T} ; \mathbb{R}^{m}\right)$, and $\left(F_{0}, f_{0}, G_{0}, g_{0}\right) \in L_{\mathcal{F}}^{2}\left(0, T ; \mathbb{R}^{m+n+m \times l+n \times d}\right)$, Equation (21) has a unique solution as $\alpha=0$. It follows from Lemma 3 that there exists a positive constant $\gamma_{0}=\gamma_{0}\left(k, \lambda, \beta_{1}, \mu_{1}, H, T\right)$ such that for any $\gamma \in\left[0, \gamma_{0}\right]$ and $\varphi \in L^{2}\left(\mathcal{F}_{T} ; \mathbb{R}^{m}\right)$, and $\left(F_{0}, f_{0}, G_{0}, g_{0}\right) \in L_{\mathcal{F}}^{2}\left(0, T ; \mathbb{R}^{m+n+m \times l+n \times d}\right)$, Equation (21) has a unique solution for $\alpha=\gamma$. Since $\gamma_{0}$ depends only on $k, \lambda, \beta_{1}, \mu_{1}, H$, $T$, we can repeat this process for $N$ times with $1 \leq N \gamma_{0}<1+\gamma_{0}$. In particular, for $\alpha=1$ with $\left(F_{0}, f_{0}, G_{0}, g_{0}\right) \equiv$ 0 , and $\varphi \equiv 0, \psi \equiv 0$, Equation (21) has a unique solution in $L_{\mathcal{F}}^{2}\left(-\delta, T ; \mathbb{R}^{n}\right) \times L_{\mathcal{F}}^{2}\left(0, T+\delta ; \mathbb{R}^{m}\right) \times$ $L_{\mathcal{F}}^{2}\left(-\delta, T ; \mathbb{R}^{n \times l}\right) \times L_{\mathcal{F}}^{2}\left(0, T+\delta ; \mathbb{R}^{m \times d}\right)$.

In the case where $m<n$ and $m=n$, our desired result can be obtained similarly. The proof of the existence of Theorem 1 is complete.

Remark 2. In the proof of the Existence of Theorem 1, (i) and (ii) in (H3) can be replaced by

(i) ${ }^{\prime} \quad \int_{0}^{T}\langle A(t, u, \alpha, \mu, \beta, v)-A(t, \bar{u}, \bar{\alpha}, \bar{\mu}, \bar{\beta}, \bar{v}), u-\bar{u}\rangle \mathrm{d} t$

$\geq \int_{0}^{T}\left[\mu_{1}\left(|H(y-\bar{y})|^{2}+|H(z-\bar{z})|^{2}\right)+\mu_{2}\left(\left|H^{\top}(p-\bar{p})\right|^{2}+\left|H^{\top}(q-\bar{q})\right|^{2}\right)\right] \mathrm{d} t$,

$\forall u=(y, p, z, q), \bar{u}=(\bar{y}, \bar{p}, \bar{z}, \bar{q}) \in \mathbb{R}^{n} \times \mathbb{R}^{m} \times \mathbb{R}^{n \times l} \times \mathbb{R}^{m \times d}, \forall t \in[0, T]$.

(ii) $^{\prime} \quad\langle\Phi(y)-\Phi(\bar{y}), H(y-\bar{y})\rangle \leq-\beta_{1}|H(y-\bar{y})|^{2}, \forall y, \bar{y} \in \mathbb{R}^{n}$.

where $\mu_{1}, \mu_{2}$ and $\beta_{1}$ are given non-negative constants with $\mu_{1}+\mu_{2}>0$ and $\mu_{2}+\beta_{1}>0$. Moreover we have $\mu_{1}>0, \beta_{1}>0$ (resp., $\left.\mu_{2}>0\right)$ when $m>n$ (resp., $\left.m<n\right)$.

\section{Conclusions}

The future evolution of a lot of processes depends not only on their current state, but also on their historical state, and these processes can usually be characterized by stochastic differential equations with time delay. In this article, we have discussed a class of differential games driven by doubly stochastic systems with time delay. To deal with the above nonzero sum differential game problem, it is natural to involve the adjoint equation, which is a kind of anticipated BDSDE. It is therefore necessary to explore a kind of general FBDSDE with the forward equation being a delayed doubly SDE and the backward equation being an anticipated BDSDE, which are so-called time-symmetry stochastic systems. This kind of FBDSDE covers a lot of the previous results, which promotes the results in [35] to doubly stochastic integrals, and extends the results in [23] to the case that involves the time delay and anticipation. We have adopted the convex variational method, and established a necessary condition and a sufficient condition for the equilibrium point of the game. In the LQ game problem, the state equation and the adjoint equation are completely coupled, then a class of linear FBDSDE is constructed, in which the forward equation is an anticipated forward doubly SDE and the backward equation is a delayed backward doubly SDE. By means of the unique solvability of the FBDSDE, the explicit expression for the Nash equilibrium point of the LQ game is obtained. Many financial and economic phenomena can be modeled by the LQ model, and we expect that the LQ game driven by doubly stochastic systems with time delay can be widely applied in these fields.

Notwithstanding that we are committed to the above game problem, we are also able to progress some consequences of optimal control for BDSDEs with time delay, for example $\mathrm{Xu}$ and Han $[19,20]$.

Author Contributions: Writing_original draft preparation, writing —review and editing, Q.Z. and H.Z.; supervision, Y.S.; Conceptualization, J.W. All authors have read and agreed to the published version of the manuscript.

Funding: This research was funded by the National Key R\&D Program of China (2018YFA0703900), National Natural Science Foundation of China $(11871309,11671229,71871129,11371226,11301298)$, Southern University of Science and Technology Start up fund (Y01286233), Natural Science Foundation of Shandong Province of China (ZR2020MA032, ZR2019MA013), Special Funds of Taishan Scholar Project (tsqn20161041), and Fostering Project of Dominant Discipline and Talent Team of Shandong Province Higher Education Institutions.

Acknowledgments: The authors express their sincerest thanks to the reviewers for their valuable comments, which further improve the conclusion and proof process of the article.

Conflicts of Interest: The authors declare no conflict of interest. 


\section{References}

1. Pardoux, P.; Peng, S.G. Backward doubly stochastic differential equations and systems of quasilinear parabolic SPDEs. Probab. Theory Relat. Fields 1994, 98, 209-227. [CrossRef]

2. Bahlali, K.; Gatt, R.; Mansouri, B.; Mtiraoui, A. Backward doubly SDEs and SPDEs with superlinear growth generators. Stoch Dynam. 2017, 17, 1-31. [CrossRef]

3. Bally, V.; Matoussi, A. Weak solutions for SPDEs and backward doubly stochastic differential equations. J. Theoret. Probab. 2001, 14, 125-164. [CrossRef]

4. Hu, L.Y.; Ren, Y. Stochastic PDIEs with nonlinear Neumann boundary conditions and generalized backward doubly stochastic differential equations driven by Lévy processes. J. Comput. Appl. Math. 2009, 229, 230-239. [CrossRef]

5. Ma, N.; Wu, Z. Backward doubly stochastic differential equations with Markov chains and a comparison theorem. Symmetry 2020, 12, 1953. [CrossRef]

6. Matoussi, A.; Piozin, L.; Popier, A. Stochastic partial differential equations with singular terminal condition. Stoch. Proc. Appl. 2017, 127, 831-876. [CrossRef]

7. Ren, Y.; Lin, A.H.; Hu, L.Y. Stochastic PDIEs and backward doubly stochastic differential equations driven by Lévy processes. J. Comput. Appl. Math. 2009, 223, 901-907. [CrossRef]

8. Shi, Y.F.; Gu, Y.L.; Liu, K. Comparison theorems of backward doubly stochastic differential equations and applications. Stoch. Anal. Appl. 2005, 23, 97-110. [CrossRef]

9. Shi, Y.F.; Wen, J.Q.; Xiong, J. Backward doubly stochastic Volterra integral equations and their applications. J. Differ. Equ. 2020, 269, 6492-6528. [CrossRef]

10. Wen, J.Q.; Shi, Y.F. Backward doubly stochastic differential equations with random coefficients and quasilinear stochastic PDEs. J. Math. Anal. Appl. 2019, 476, 86-100. [CrossRef]

11. Wu, Z.; Zhang, F. BDSDEs with locally monotone coefficients and Sobolev solutions for SPDEs. J. Differ. Equ. 2011, 251, 759-784. [CrossRef]

12. Zhang, Q.; Zhao, H.Z. Stationary solutions of SPDEs and infinite horizon BDSDEs. J. Funct. Anal. 2007, 252, 171-219. [CrossRef]

13. Zhang, Q.; Zhao, H.Z. Stationary solutions of SPDEs and infinite horizon BDSDEs under non-Lipschitz coefficients. J. Differ. Equ. 2010, 248, 953-991. [CrossRef]

14. Zhang, Q.; Zhao, H.Z. SPDEs with polynomial growth coefficients and the Malliavin calculus method. Stoch. Proc. Appl. 2013, 123, 2228-2271. [CrossRef]

15. Zhang, Q.; Zhao, H.Z. Backward doubly stochastic differential equations with polynomial growth coefficients. Discrete Con. Dyn. A 2015, 35, 5285-5315. [CrossRef]

16. Bahlali, S.; Gherbal, B. Optimality conditions of controlled backward doubly stochastic differential equations. Random Oper. Stoch. Equ. 2010, 18, 247-265. [CrossRef]

17. Han, Y.C.; Peng, S.G.; Wu, Z. Maximum principle for backward doubly stochastic control systems with applications. SIAM J. Control Optim. 2010, 48, 4224-4241. [CrossRef]

18. Wu, J.B.; Liu, Z.M. Optimal control of mean-field backward doubly stochastic systems driven by Itô-Lévy processes. Int. J. Control 2020, 93, 953-970. [CrossRef]

19. Xu, J.; Han, Y.C. Stochastic maximum principle for delayed backward doubly stochastic control systems. J. Nonlinear Sci. Appl. 2017, 10, 215-226. [CrossRef]

20. $\mathrm{Xu}, \mathrm{J}$. Stochastic maximum principle for delayed doubly stochastic control systems and their applications. Int. J. Control 2020, 93, 1371-1380. [CrossRef]

21. Zhu, Q.F.; Shi, Y.F. Optimal control of backward doubly stochastic systems with partial information. IEEE Trans. Autom. Control 2015, 60, 173-178. [CrossRef]

22. Peng, S.G.; Shi, Y.F. A type of time-symmetric forward-backward stochastic differential equations. C. R. Acad. Sci. Paris Ser. I 2003, 336, 773-778. [CrossRef]

23. Zhu, Q.F.; Shi, Y.F.; Gong, X.J. Solutions to general forward-backward doubly stochastic differential equations. Appl. Math. Mech. 2009, 30, 517-526. [CrossRef]

24. Zhu, Q.F.; Shi, Y.F. Forward-backward doubly stochastic differential equations and related stochastic partial differential equations. Sci. China Math. 2012, 55, 2517-2534. [CrossRef]

25. Zhang, L.Q.; Shi, Y.F. Maximum principle for forward-backward doubly stochastic control systems and applications. ESAIM Control Optim. Calc. Var. 2011, 17, 1174-1197. [CrossRef]

26. Shi, Y.F.; Zhu, Q.F. Partially observed optimal control of forward-backward doubly stochastic systems. ESAIM Control Optim. Calc. Var. 2013, 19, 828-843. [CrossRef]

27. Von Neumann, J.; Morgenstern, O. The Theory of Games and Economic Behavior; Princeton University Press: Princeton, NJ, USA, 1944.

28. Nash, J. Non-cooperative games. Ann. Math. 1951, 54, 286-295. [CrossRef]

29. Zhao, H.; Shen, Y.; Zeng, Y. Time-consistent investment-reinsurance strategy for mean-variance insurers with a defaultable security. J. Math. Anal. Appl. 2016, 437, 1036-1057. [CrossRef]

30. Wang, G.C.; Yu, Z.Y. A Pontryagin's maximum principle for nonzero sum differential games of BSDEs with applications. IEEE Trans. Autom. Control 2010, 55, 1742-1747. [CrossRef] 
31. Wang, G.C.; Yu, Z.Y. A partial information non-zero sum differential game of backward stochastic differential equations with applications. Automatica 2012, 48, 342-352. [CrossRef]

32. Yu, Z.Y.; Ji, S.L. Linear-quadratic non-zero sum differential game of backward stochstic differential equations. In Proceedings of the 27th Chinese Control Conference, Kunming, China, 16-18 July 2008; pp. 562-566.

33. Hui, E.; Xiao, H. Differential games of partial information forward-backward doubly SDE and applications. ESAIM Control Optim. Calc. Var. 2014, 20, 78-94. [CrossRef]

34. Chen, L.; Wu, Z. Maximum principle for the stochastic optimal control problem with delay and application. Automatica 2010, 46, 1074-1080. [CrossRef]

35. Chen, L.; Wu, Z. A type of generalized forward-backward stochastic differential equations and applications. Chin. Ann. Math. Ser. B 2011, 32, 279-292. [CrossRef]

36. Chen, L.; Huang, J.H. Stochastic maximum principle for controlled backward delayed system via advanced stochastic differential equation. J. Optim. Theory Appl. 2015, 167, 1112-1135. [CrossRef]

37. Meng, Q.X.; Shen, Y. Optimal control of mean-field jump-diffusion systems with delay: A stochastic maximum principle approach. J. Comput. Appl. Math. 2015, 279, 13-30. [CrossRef]

38. Meng, Q.X.; Shen, Y. Optimal control for stochastic delay evolution equations. Appl. Math. Optim. 2016, 74, 53-89. [CrossRef]

39. Shen, Y.; Meng, Q.X.; Shi, P. Maximum principle for jump-diffusion mean-field stochastic delay differential equations and its application to finance. Automatica 2014, 50, 1565-1579. [CrossRef]

40. Chen, L.; Yu, Z.Y. Maximum principle for nonzero-sum stochastic differential game with delays. IEEE Trans. Autom. Control 2015, 60, 1422-1426. [CrossRef]

41. Shi, J.T.; Wang, G.C. A nonzero sum differential game of BSDE with time-delayed generator and applications. IEEE Trans. Autom. Control 2016, 61, 1959-1964. [CrossRef]

42. Shen, Y.; Zeng Y. Optimal investment-reinsurance with delay for mean-variance insurers: A maximum principle approach. Insur. Math. Econ. 2014, 57, 1-12. [CrossRef]

43. Arriojas, M.; Hu, Y.Z.; Mohammed, S.E.; Pap, G. A delayed black and scholes formula. Stoch. Anal. Appl. 2007, 25, 471-492. [CrossRef]

44. Kazmerchuk, Y.; Swishchuk, A.; Wu, J.H. The pricing of option for securities markets with delayed response. Math. Comput. Simul. 2007, 75, 69-79. [CrossRef]

45. Xu, X.M. Anticipated backward doubly stochastic differential equations. Appl. Math. Comput. 2013, 220, 53-62. [CrossRef]

46. Zhang, F. Anticipated backward doubly stochastic differential equations. Sci. Sin. Math. 2013, 43, 1223-1236. (In Chinese) [CrossRef]

47. Peng, S.G.; Wu, Z. Fully coupled forward-backward stochastic differential equations and applications to optimal control. SIAM J. Control Optim. 1999, 37, 825-843. [CrossRef]

48. Zhu, Q.F.; Shi, Y.F. Nonzero-sum differential game of backward doubly stochastic systems with delay and applications. Math. Control Relat. F 2021, 11, 73-94. [CrossRef]

49. Peng, S.G. Probabilistic interpretation for systems of quasilinear parabolic partial differential equations. Stochastics 1991, 37, 61-74. 